\title{
JUMPS OF ORDERINGS
}

\author{
C. J. ASH, C. G. JOCKUSCH, JR., AND J. F. KNIGHT
}

\begin{abstract}
Here it is shown that for each recursive ordinal $\alpha \geq 2$ and each Turing degree $\mathbf{d}>\mathbf{0}^{(\alpha)}$, there is a linear ordering $\mathbf{A}$ such that $\mathbf{d}$ is least among the $\alpha$ th jumps of degrees of (open diagrams of) isomorphic copies of $\mathbf{A}$ and for $\beta<\alpha$, the set of $\beta$ th jumps of degrees of copies of $\mathbf{A}$ has no least element.
\end{abstract}

\section{INTRODUCTION}

This paper improves the results of $\left[\mathrm{K}_{2}\right]$ by showing that for each recursive ordinal $\alpha \geq 2$, there is a linear ordering $\mathbf{A}_{\alpha}$ such that $\mathbf{A}_{\alpha}$ has $\alpha$ th jump degree and does not have $\beta$ th jump degree for any $\beta<\alpha$. All structures to be considered here have universe $\omega$, and all languages are recursive. Using an appropriate Gödel numbering, we can, for an arbitrary structure $\mathbf{A}$, think of the open diagram $D(\mathbf{A})$ as a subset of $\omega$. We write $\operatorname{deg}(S)$ for the Turing degree of a set $S \subseteq \omega$. For any structure $\mathbf{A}, \operatorname{deg}(D(\mathbf{A}))$ provides some measure of the complexity of A. A slightly unpleasant feature of this measure is that it depends on the presentation as well as the isomorphism type of $\mathbf{A}$. Let $\operatorname{Dg}(\mathbf{A})=\{\operatorname{deg}(D(\mathbf{B})): \mathbf{B} \cong \mathbf{A}\}$. Unless $\mathbf{A}$ is "trivial" in the sense of $\left[\mathrm{K}_{2}\right]$, the set $\operatorname{Dg}(\mathbf{A})$ is closed upwards. No linear ordering is trivial. (We are not counting finite orderings as structures since they do not have universe $\omega$.)

It would be satisfying to assign Turing degrees to isomorphism types of structures, and for certain types of structures we can do this by considering a simplest presentation. A structure $\mathbf{A}$ is said to have degree $\mathbf{d}$ if $\mathbf{d}$ is the least element of the set $\operatorname{Dg}(\mathbf{A})$. Many structures fail to have a degree. There are many orderings with no recursive copy, and Richter [Ri] showed that no such ordering has a degree. (If an ordering has degree $\mathbf{d}$, then $\mathbf{d}=\mathbf{0}$.)

By considering jumps of presentations, we can assign Turing degrees to some isomorphism types of structures that do not have a degree. For any set $X \subseteq \omega$, the jump of $X$ is $X^{\prime}=\left\{e: \varphi_{e}^{X}(e)\right.$ converges $\}$. The jump operation can be iterated to form $X^{(\alpha)}$ for any recursive ordinal $\alpha$ (see [Ro]). The choice of a set $X^{(\alpha)}$ depends on the ordinal notation for $\alpha$, although the Turing degree

Received by the editors May 12, 1988 and, in revised form, August 15, 1988.

1980 Mathematics Subject Classification (1985 Revision). Primary 03C57, 03D45; Secondary 06A05.

The authors were partially supported by N.S.F. grants DMS 85-03353 (Ash), DMS 86-01242 (Jockusch), and DMS 85-03353 and DMS 87-01559 (Knight). 
is independent of the notation. The idea is to let $X^{(\alpha+1)}=\left(X^{(\alpha)}\right)^{\prime}$, and, for limit $\alpha$, to let $X^{(\alpha)}=\left\{(n, k): k \in X^{\left(\alpha_{n}\right)}\right\}$, where $\alpha_{n}$ is the $n$th member of the fundamental sequence (increasing and converging to $\alpha$ ) that is picked out by the notation for $\alpha$. Now, we can say what it means for a structure to have 2 nd or $\omega$ th jump degree. For any structure $\mathbf{A}$, and any recursive ordinal $\alpha$, we say that the structure $\mathbf{A}$ has $\alpha$ th jump degree $\mathbf{d}$ if $\mathbf{d}$ is the least element of the set $\left\{\mathbf{b}^{(\alpha)}: \mathbf{b} \in \operatorname{Dg}(\mathbf{A})\right\}$.

In $\left[K_{2}\right]$ it was shown that if an ordering has 1 st jump degree $\mathbf{d}$, then $\mathbf{d}=\mathbf{0}^{\prime}$. Recently, Jockusch and Soare [JS] have shown that there is an ordering $\mathbf{A}$ that has 1 st jump degree $\mathbf{0}^{\prime}$ and does not have a degree. In fact, for any nonrecursive r.e. set $C$, they can produce an ordering $\mathbf{A}$ such that $D(\mathbf{A}) \leq_{T} C$ and for all $\mathbf{B} \cong \mathbf{A}, D(\mathbf{B})$ is not recursive. In $\left[\mathrm{K}_{2}\right]$ there are examples of structures $\mathbf{C}_{m}$, for $0 \leq m<\omega$, such that $\boldsymbol{C}_{m}$ has $2 m+2$ nd jump degree and does not have $m+1$ st jump degree. Moreover, the $2 m+2$ nd jump degree can be any degree d $>\mathbf{0}^{(2 m+2)}$. Ash and Knight have analyzed further the examples from $\left[\mathrm{K}_{2}\right]$ and can show that $\mathbf{C}_{m}$ does not have $2 m+1$ st jump degree. Hence, for each even integer $n \geq 2$, there are orderings having $n$th jump degree and not having $n-1$ st jump degree.

In the present paper we show that for each recursive ordinal $\alpha \geq 2$ and each degree $\mathbf{d}>\mathbf{0}^{(\alpha)}$, there is an ordering $\mathbf{A}_{\alpha}$ such that $\mathbf{A}_{\alpha}$ has $\alpha$ th jump degree $\mathbf{d}$ and $\mathbf{A}_{\alpha}$ does not have $\beta$ th jump degree for any $\beta<\alpha$. For $\alpha$ an even integer, the examples given here differ from those in $\left[\mathrm{K}_{2}\right]$. The method used here for analyzing the orderings gives precise characterizations of sets $\operatorname{Dg}\left(\mathbf{A}_{\alpha}\right)$, while the method in $\left[\mathrm{K}_{2}\right]$ does not. We shall describe a general procedure for encoding an arbitrary set $S \subseteq \omega$ in orderings $\mathbf{A}_{\alpha}(S)$ for all recursive ordinals $\alpha$. (Actually, there will be orderings for all ordinal notations $a \in O$.) We characterize $\operatorname{Dg}\left(\mathbf{A}_{\alpha}(S)\right)$ for all $S$ and $\alpha$. Once we have done this, we choose sets $S$ with some special properties and let $\mathbf{A}_{\alpha}=\mathbf{A}_{\alpha}(S)$.

In $\S 1$, we establish the existence of sets $S$ with the special properties needed for the orderings $\mathbf{A}_{\alpha}$. In $\S 2$, we begin by describing a basic ordering $\sigma^{*}(S)$ that encodes a set $S$, and we characterize $\operatorname{Dg}\left(\sigma^{*}(S)\right)$ as the set $\{\operatorname{deg}(D): S$ is r.e. in $\left.D^{\prime \prime}\right\}$. (We shall take $\mathbf{A}_{2}(S)$ and $\mathbf{A}_{3}(S)$ to be $\sigma^{*}(S \oplus(\omega-S)$ ) and $\sigma^{*}(S)$, respectively.) Next, we characterize $\operatorname{Dg}(Z \cdot \mathbf{A})$ in terms of $\operatorname{Dg}(\mathbf{A})$, for an arbitrary ordering $\mathbf{A}$. Having done this, we define $\mathbf{A}_{n}(S)$ for all finite $n \geq 2$. (We let $\mathbf{A}_{4}(S)=Z \cdot \mathbf{A}_{2}(S), \mathbf{A}_{5}(S)=Z \cdot \mathbf{A}_{3}(S)$, and in general for $n \geq 2$, $\mathbf{A}_{n+2}(S)=Z \cdot \mathbf{A}_{n}(S)$.) We show that for appropriately chosen sets $S, \mathbf{A}_{n}(S)$ is the desired $\mathbf{A}_{n}$ having $n$th jump degree and not having $n-1$ st jump degree.

In $\S 3$, we give a relatively simple procedure for combining the orderings from $\S 2$ to form an ordering that has $\omega$ th jump degree and does not have $n$th jump degree for any $n<\omega$. (Later, we shall obtain a slightly different ordering $\mathbf{A}_{\omega}$ from the general procedure that we develop for producing $\mathbf{A}_{\alpha}$ for arbitrary recursive limit ordinals $\alpha$.) Also in $\S 3$, we describe a new kind of ordering $\nu^{\omega}(S)$ that encodes a set $S$, and we characterize $\operatorname{Dg}\left(\nu^{\omega}(S)\right)$. (We shall take 
$\mathbf{A}_{\omega+1}(S)$ and $\mathbf{A}_{\omega+2}(S)$ to be $\nu^{\omega}(S \oplus(\omega-S))$ and $\nu^{\omega}(S)$, respectively.

In $\S 4$, we define $\nu^{\alpha}(S)$ for arbitrary limit ordinals $\alpha$ by analogy with the special case $\nu^{\omega}(S)$. We then define $\mathbf{A}_{\alpha}(S)$ for all recursive ordinals $\alpha \geq 2$. There are still some lemmas to prove. One is a general result on combining sequences of orderings, which will be used in connection with $\mathbf{A}_{\alpha}(S)$ for recursive limit $\alpha$. Another is an extension of the lemma from $\S 3$ characterizing $\operatorname{Dg}\left(\nu^{\alpha}(S)\right)$ for arbitrary recursive limit $\alpha$. There is also an extension of one of the lemmas from $\S 2$ characterizing $\operatorname{Dg}\left(Z^{\alpha} \cdot \mathbf{A}\right)$ in terms of $\operatorname{Dg}(\mathbf{A})$, for an arbitrary ordering $\mathbf{A}$ and an arbitrary recursive ordinal $\alpha$. Equipped with these lemmas, we characterize $\operatorname{Dg}\left(\mathbf{A}_{\alpha}(S)\right)$ for all $S \subseteq \omega$ and all recursive ordinals $\alpha \geq 2$. Finally, we apply the lemmas from $\S 1$ to obtain sets $S$ such that $\mathbf{A}_{\alpha}(S)$ is the desired ordering $\mathbf{A}_{\alpha}$ having $\alpha$ th jump degree and not having $\beta$ th jump degree for any $\beta<\alpha$.

Most of our terminology and notation is standard. We mention one piece here. In analyzing our orderings, we shall use some equivalence relations. For any ordering $\mathbf{A}$, we define relations $\sim_{\alpha}$, for all ordinals $\alpha$, as follows: $a \sim_{0} b$ if the interval between $a$ and $b$ is finite, $a \sim_{\alpha+1} b$ if the interval between $a$ and $b$ has only finitely many $\sim_{\alpha}$-classes, and for $\alpha$ a limit ordinal, $a \sim_{\alpha} b$ if there is some $\beta<\alpha$ such that $a \sim_{\beta} b$.

\section{BASIC RECURSION-THEORETIC FACTS}

In this section, we establish the existence of sets with some special recursiontheoretic properties. The sets will be generic, and we begin by describing briefly the kind of forcing used to produce them. First, let $L$ be a language with a unary predicate $D$ (for the generic set) in addition to the usual symbols used in arithmetic, $+, \cdot,<$, and a constant $\underline{n}$ for each $n \in \omega$. We consider recursive infinitary $L$-formulas. For recursive ordinals $\alpha$, the recursive infinitary $\Sigma_{\alpha}$ and $\Pi_{\alpha}$ formulas are as follows:

(1) The recursive infinitary $\Sigma_{0}$ and $\Pi_{0}$ formulas are just the finitary open formulas.

(2) The recursive infinitary $\Sigma_{\alpha+1}$ formulas $\varphi(\vec{x})$ are disjunctions of r.e. sets of formulas of the form $\exists \vec{y} \psi(\vec{x}, \vec{y})$, where $\psi$ is recursive infinitary $\Pi_{\alpha}$, and the recursive infinitary $\Pi_{\alpha+1}$ formulas $\varphi(\vec{x})$ are conjunctions of r.e. sets of formulas of the form $\forall \vec{y} \psi(\vec{x}, \vec{y})$, where $\psi$ is recursive infinitary $\Sigma_{\alpha}$.

(3) For limit $\alpha$, the recursive infinitary $\Sigma_{\alpha}$ formulas $\varphi(x)$ are disjunctions of r.e. sets of recursive infinitary $\Sigma_{\beta}$ and $\Pi_{\beta}$ formulas $\psi(\vec{x})$ for $\beta<\alpha$, and the recursive infinitary $\Pi_{\alpha}$ formulas $\varphi(\vec{x})$ are conjunctions of r.e. sets of recursive infinitary $\Sigma_{\beta}$ and $\Pi_{\beta}$ formulas $\psi(\vec{x})$ for $\beta<\alpha$.

To be more precise, we should develop the hierarchy of recursive infinitary formulas simultaneously with a system of indices for the formulas, referring to ordinal notations $a \in O$. (For a general discussion of ordinal notations and definitions of $O$ and $<_{O}$ see [Ro].) Let $\vec{y}_{i}$ denote the $i$ th finite sequence 
of variables in some fixed recursive enumeration. Let $(\forall \vec{y})_{i}$ be the block of universal quantifiers corresponding to the sequence $\vec{y}_{i}$. If $a \in O$ is a notation for $\alpha$, where $\alpha \neq 0$, then for each $e$ and each finite sequence of variables $\vec{x}$, $(\Sigma, e, a, \vec{x})$ is a notation for the recursive $\Sigma_{\alpha}$ formula $\varphi(\vec{x})$, with free variables included in $\vec{x}$, that is given by $W_{i \in S_{e, a}}(\forall \vec{y})_{(i)_{0}} \psi_{(i)_{1}}\left(\vec{x}, \vec{y}_{(i)_{0}}\right)$, where $S_{e, a}$ is the set of all $i \in W_{e}$ such that $(i)_{1}$ is an index of the form $\left(\Pi, e^{\prime}, b, \vec{x}^{\wedge} \vec{y}_{(i)_{0}}\right)$ for some $b<_{O} a$ (we wrote $\psi_{(i)_{1}}$ above for the subformula of $\varphi$ having this index.) Similarly, we have indices $(\Pi, e, a, \vec{x})$ for recursive $\Pi_{\alpha}$ formulas. We shall say that the set of recursive infinitary formulas of complexity at most $\alpha$ is r.e. What we mean by this is that for whatever notation $a$ for $\alpha$ that we have in mind, there is an r.e. set consisting of all indices of the forms $(\varepsilon, e, b, \vec{x})$ and $(\Pi, e, b, \vec{x})$ with $b<_{o} a$ or $b=a$. (See [A] for more on recursive infinitary formulas.)

In producing a generic subset of $\omega$, we use the elements of $2^{<\omega}$ as forcing conditions. Let $\mathbf{N}$ denote the standard model of arithmetic. Then we define forcing as follows:

(1) If $\varphi$ is a finitary open sentence, then $p \Vdash \varphi$ iff $n \in \operatorname{dom}(p)$ for all constants $\underline{n}$ appearing in $\varphi$, and $(\mathbf{N}, S) \vDash \varphi$ for all $S$ (or, equivalently, for some $S)$ such that $\chi_{S} \supseteq p$.

(2) If $\varphi$ has the form $\exists x \psi(x)$, then $p \Vdash \varphi$ iff $p \Vdash \psi(\underline{n})$ for some $n \in \omega$.

(3) If $\varphi$ has the form $\forall x \psi(x)$, then $p \Vdash \varphi$ iff for all $\underline{n}$ and all $q \supseteq p$, there is some $r \supseteq q$ such that $r \Vdash \Psi(\underline{n})$.

(4) If $\varphi$ is the disjunction of an r.e. set of sentences $\psi_{n}$, then $p \Vdash \varphi$ iff $p \Vdash \psi_{n}$ for some $n$.

(5) $\hat{\mathbb{i}} \hat{i} \varphi$ is the conjunction of an r.e. set of sentences $\psi_{n}$, but is not a finitary open sentence, then $p \Vdash \varphi$ iff for each $n$ and each $q \supseteq p$, there is some $r \supseteq q$ such that $r \Vdash \psi_{n}$.

(5) If $r$ has the form $\sim \psi$, where $\psi$ is not a finitary open sentence, then $p \Vdash \varphi$ iff there is no $q \supseteq p$ such that $q \Vdash \psi$.

Th: relations $p \Vdash k \in D^{(\alpha)}$ and $p \Vdash k \notin D^{(\alpha)}$, on $p$ and $k$, are recursive in $0^{(\alpha)}$. Forcing for finitary open sentences is recursive, even for negations. Note that $p$ will force the negation of a $\Sigma_{\beta}$ sentence iff it forces the dual $\Pi_{\beta}$ sentence that is equivalent to the negation. For a recursive infinitary formula $\varphi$, the arithmetical formula saying in $\mathbf{N}$ that $p \Vdash \varphi$ is itself $\Sigma_{\alpha}$ if $\varphi$ is $\Sigma_{\alpha}$, and is $\Pi_{\alpha}$ if $\varphi$ is $\Pi_{\alpha}$. A set $S \subseteq \omega$ is $\alpha$-generic if $\chi_{S}$ is the union of a chain of forcing conditions $\left(p_{n}\right)_{n \in \omega}$ such that for each infinitary recursive $L$-sentence $\varphi$ that is either $\Sigma_{\beta}$ or $\prod_{\beta}$ for some $\beta \leq \alpha$, there is some $n$ such that $p_{n}$ decides $\varphi$. (Actually, we have in mind a particular notation $a \in O$ for $\alpha$, and the sentences $\varphi$ that must be decided are those with indices $(\Sigma, e, b, \varnothing)$ and $(\Pi, e, b, \varnothing)$ for $b<_{O} a$ or $b=a$.) It is easy to check that the usual facts about the existence of generic sets and the relation between truth and forcing all hold here. With this much general discussion, we begin on the lemmas. 
The first lemma is a transfinitely iterated Jump Inversion Theorem due to J. Macintyre (see [Mac, Theorem 2]). The proof uses forcing. Since our forcing language is slightly different from Macintyre's, and we shall use a modification of the proof later, we give a sketch here.

Lemma 1.1. For any recursive ordinal $\alpha$ and any $A$ such that $0^{(\alpha)} \leq_{T} A$, there exists an $\alpha$-generic set $S$ such that $S \oplus 0^{(\alpha)} \equiv_{T} S^{(\alpha)} \equiv_{T} A$.

Proof. The sequence of forcing conditions $\left(p_{n}\right)_{n \in \omega}$ that determines $S$ will have a special feature. We fix a recursive enumeration of the sentences of complexity at most $\alpha$. (We have in mind a notation for $\alpha$, and we explained above the sense in which the set of sentences of complexity at most $\alpha$ is r.e.) Let $p_{0}=\varnothing$. Given $p_{n}$, let $q$ be the first extension of $p_{n}$ that decides the $n$th sentence on our list. Then (in order to help encode $A$ ) let $p_{n+1}$ be $q^{\wedge} 1$ if $n \in A$ and $q^{\wedge} 0$ if $n \notin A$. We must show that the $\alpha$-generic set $S$ obtained from the sequence $\left(p_{n}\right)_{n \in \omega}$ has the desired properties.

Trivially, we have $0^{(\alpha)} \oplus S \leq_{T} S^{(\alpha)}$. We must show that $S^{(\alpha)} \leq_{T} 0^{(\alpha)} \oplus S$. To decide whether $k \in S^{(\alpha)}$, we look for a finite $p \subseteq \chi_{S}$ such that $p \Vdash k \in D^{(\alpha)}$ or $p \Vdash k \notin D^{(\alpha)}$. Knowing $S$, we can check whether $p \subseteq \chi_{S}$, and knowing $0^{(\alpha)}$, we can check whether $p \Vdash k \in D^{(\alpha)}$ or $p \Vdash k \notin D^{(\alpha)}$. This shows that $S^{(\alpha)} \leq_{T} 0^{(\alpha)} \oplus S$. Therefore, $0^{(\alpha)} \oplus S \equiv_{T} S^{(\alpha)}$. We must also show that $S^{(\alpha)} \equiv_{T} A$. Since the whole construction is recursive in $A$, we have $S^{(\alpha)} \leq_{T} A$. The set $A$ is recursive in the sequence $\left(p_{n}\right)_{n \in \omega}$ (encoded in the last terms of the $p_{n}$ 's). The sequence $\left(p_{n}\right)_{n \in \omega}$ is recursive in $0^{(\alpha)}$ and $S$. (Having found $p_{n}$, we locate $p_{n+1}$ by finding the first $q \supseteq p_{n}$ such that $q$ decides the $n$th sentence of the appropriate form and then checking to see which of $q^{\wedge} 0, q^{\wedge} 1$ is an initial segment of $\chi_{S}$.) Therefore, $A \leq_{T} 0^{(\alpha)} \oplus S$, so we have $S^{(\alpha)} \equiv_{T} 0^{(\alpha)} \oplus S \equiv_{T} A$. This completes the proof of Lemma 1.1.

The next two lemmas will be important in showing that our orderings $\mathbf{A}_{\alpha+1}$ do not have $\alpha$ th jump degree. Lemma 1.2 is the more difficult of the two.

Lemma 1.2. Let $\alpha$ be a recursive ordinal, and let $S \subseteq \omega$ be $\alpha+1$-generic. If $\mathscr{C}=\left\{D: S\right.$ is r.e. in $\left.D^{(\alpha)}\right\}$, then for any $B \subseteq \omega$ such that $B \leq_{T} D^{(\alpha)}$ for all $D \in \mathscr{C}$, we have $B \leq_{T} 0^{(\alpha)}$. Hence, since $S$ is not r.e. in $0^{(\alpha)}$, the set $\left\{D^{(\alpha)}: D \in \mathscr{C}\right\}$ has no element of least degree.

Proof. Suppose that $B$ is not recursive in $0^{(\alpha)}$. We must find $D \in \mathscr{C}$ such that $B$ is not recursive in $D^{(\alpha)}$. We shall produce a set $A$ such that $S$ is r.e. in $A$ and for all $e, \varphi_{e}^{A, 0^{(n)}} \neq \chi_{B}$. Suppose for the moment that there is such a set $A$. Applying Lemma 1.1, we obtain $D$ such that $D^{(\alpha)} \equiv_{T} 0^{(\alpha)} \oplus A$. Then $D$ is the set we want, with $S$ r.e. in $D^{(\alpha)}$ and $B$ not recursive in $D^{(\alpha)}$. We shall construct $A \subseteq \omega \times \omega$ such that $i \in S$ iff $(i, j) \in A$ for some $j$. This will guarantee that $S$ is r.e. in $A$. We let $\mathscr{F}$ be the set of all $0-1$ valued functions whose domains are finite subsets of $\omega \times \omega$. For each $\sigma \in \mathscr{F}$, let 
$S_{\sigma}=\{i: \exists j(\sigma(i, j)=1)\}$. We say that $\sigma$ is admissible if $S_{\sigma} \subseteq S$. We shall form $\chi_{A}$ as the union of a chain of admissible functions. Clearly, for any admissible $\sigma$ and any $i \in S$, there is an admissible $\tau \supseteq \sigma$ with $i \in S_{\tau}$. The existence of the desired set $A$ reduces to the following.

Claim. For any admissible $\sigma$ and any $e \in \omega$, there is an admissible $\tau \supseteq \sigma$ such that one of the following holds for some $k:(1) \varphi_{e}^{\tau, 0^{(m)}}(k)$ converges with value $t \neq \chi_{B}(k),(2)$ there is no admissible $\rho \supseteq \tau$ such that $\varphi_{e}^{\rho, 0^{(n)}}(k)$ converges.

Proof of Claim. Suppose the claim is false; i.e., for some admissible $\sigma$ and some $e$, (1) and (2) both fail for all admissible $\tau \supseteq \sigma$ and all $k$. We say that $\tau_{1}, \tau_{2} \in \mathscr{F}$ (not necessarily admissible) $e$-split if there is some $k \in \omega$ such that $\varphi_{e}^{\tau_{i}, 0^{(m)}}(k)=t_{i}$, where $t_{1} \neq t_{2}$. Let $\mathscr{K}$ be the family of finite sets $S_{\tau_{1}} \cup S_{\tau_{2}}$ such that $\tau_{1}, \tau_{2} e$-split, where $\tau_{1}, \tau_{2}$ are extensions of $\sigma$ in $\mathscr{F}$. Note that if $E \in \mathscr{K}$, then $E \cap(\omega-S) \neq \varnothing$. Otherwise, we would have an admissible $\tau \supseteq \sigma$ and $k$ satisfying (2). The sentence in our forcing language saying of the $\alpha+1$-generic set $S$ that $(\forall E \in \mathscr{K})(E \cap(\omega-S) \neq \varnothing)$ is a $\Pi_{\alpha+1}$ sentence (since $\mathscr{K}$, coded as a set of natural numbers, is defined by a $\Sigma_{\alpha}$ formula). Since the sentence is true, it must be forced by some finite $\mu \subseteq \chi_{S}$. Then there is some $k_{0}$ such that for all $E \in \mathscr{K}, E \cap(\omega-S) \cap k_{0} \neq \varnothing$.

We shall obtain a contradiction by considering an admissible $\tau \supset \sigma$ such that $S_{\tau} \cap k_{0}=S \cap k_{0}$. By our assumption, for each $k$, there is not admissible $\rho \supseteq \tau$ such that $\varphi_{e}^{\rho, 0^{(n)}}(k)$ converges with value $t \neq \chi_{B}(k)$, and there is some admissible $\rho \supseteq \tau$ such that $\varphi_{e}^{\rho, 0^{(n)}}(k)$ converges. It follows from this that $B \leq_{T} 0^{(\alpha)}$. To decide whether $k \in B$, look for $\rho \in \mathscr{F}$, not necessarily admissible, such that $\rho \supseteq \tau, S_{\rho} \cap k_{0}=S \cap k_{0}$, and $\varphi_{e}^{\rho, 0^{(a)}}(k)$ converges. The only possible value is $\chi_{B}(k)$. This is a contradiction, so the claim is proved. Now, it is clear that we can obtain the set $D$ to complete the proof of the lemma.

The next lemma is surely known to anyone who ever wondered whether it was true.

Lemma 1.3. Let $\alpha$ be a recursive ordinal. Let $\mathscr{C}=\left\{D: S \leq_{T} D^{(\alpha+1)}\right\}$, for some set $S$. If $B \leq_{T} D^{(\alpha)}$, for all $D \in \mathscr{C}$, then $B \leq_{T} 0^{(\alpha)}$. Hence, if $S$ is not recursive in $0^{(\alpha+1)}$, then $\left\{D^{(\alpha)}: D \in \mathscr{C}\right\}$ has no element of least degree.

Proof. Fix $S$ and $B$, where $B$ is not recursive in $0^{(\alpha)}$. We must show that there is some $D \in \mathscr{C}$ such that $B$ is not recursive in $D^{(\alpha)}$. We shall construct a set $A$ such that $S \leq_{T} A \oplus 0^{(\alpha+1)}$ and $B$ is not recursive in $A \oplus 0^{(\alpha)}$. Suppose for the moment that we have $A$. Then applying Lemma 1.1, we get $D$ with $D^{(\alpha)} \equiv_{T} A \oplus 0^{(\alpha)}$. Since $S \leq_{T} A \oplus 0^{(\alpha+1)} \leq_{T}\left(A \oplus 0^{(\alpha)}\right)^{\prime} \equiv_{T}\left(D^{(\alpha)}\right)^{\prime} \equiv_{T} D^{(\alpha+1)}$, 
we have $D \in \mathscr{C}$ with $B$ not recursive in $D^{(\alpha)}$. Therefore, constructing $A$ will complete the proof of the lemma.

To construct $A$, we form a tree $\mathscr{T}=\bigcup_{n \in \omega} \mathscr{T}_{n}$. Let $\mathscr{T}_{0}=\{\varnothing\}$. Given $\mathscr{T}_{m}$, take each $\sigma \in \mathscr{T}_{m}$, and find, if possible, two extensions $\tau_{1}, \tau_{2}$ such that for some $k, \varphi_{m}^{\tau_{i}, 0^{(i)}}(k)$ converges with value $t_{i}$, where $t_{1} \neq t_{2}$. We again say that $\tau_{1}, \tau_{2} m$-split. Then put $\tau_{i}^{\wedge} 0$ and $\tau_{i}^{\wedge} 1$ into $\mathscr{T}_{m+1}$. If $\sigma$ does not have a pair of extensions that $m$-split, then simply put $\sigma^{\wedge} 0$ and $\sigma^{\wedge} 1$ into $\mathscr{T}_{m+1}$. The tree formed this way is recursive in $0^{(\alpha+1)}$. Now, we define $A$ by letting $\chi_{A}$ be the union of a path $\left(\sigma_{m}\right)_{m \in \omega}$ in $\mathscr{T}$ such that if $\sigma_{m}$ has $m$-splitting extensions, then for some $k, \varphi_{m}^{\sigma_{m+1}, 0^{(x)}}(k)$ converges with value $t \neq \chi_{B}(k)$, and the last term of $\sigma_{m+1}$ is 1 if $m \in S$ and 0 if $m \notin S$. Clearly, $S \leq_{T} A \oplus 0^{(\alpha+1)}$. We must show that $B$ is not recursive in $A \oplus 0^{(\alpha)}$. Suppose that $\varphi_{e}^{A, 0^{(\alpha)}}=\chi_{B}$. There must not have been $e$-splitting. This means that we can decide whether $k \in B$ by searching for $\tau \supseteq \sigma_{e}$ such that $\varphi_{e}^{\tau, 0^{(x)}}(k)$ converges, say with value $t$. We know that there is some $\tau$ which gives convergence and the only possible value for $t$ is $\chi_{B}(k)$. This contradicts the assumption that $B$ is not recursive in $0^{(\alpha)}$. Therefore, we have the desired $A$, and the proof is complete.

The next lemma is important in showing that for recursive limit ordinals $\alpha$, $\mathbf{A}_{\alpha}$ does not have $\beta$ th jump degree for any $\beta<\alpha$. The original proof used model theory although the present proof does not.

Lemma 1.4. Let $\alpha$ be a recursive limit ordinal, and let $\left(\alpha_{n}\right)_{n \in \omega}$ be the fundamental sequence with limit $\alpha$ that is picked out by a notation for $\alpha$. Let $S \subseteq \omega \times \omega$, where $S_{n}=\{k:(n, k) \in S\}$. Let $\mathscr{C}=\left\{D: S_{n} \leq_{T} D^{\left(\alpha_{n}\right)}\right.$ uniformly in $n\}$, and suppose that $B \leq_{T} D^{(\beta)}$ for all $D \in \mathscr{C}$. If $\alpha_{k}>\beta$, and $T_{k}=\bigoplus_{n<k} S_{n}$, then $B \leq_{T} T_{k}^{(\beta)}$. Hence, if $\beta<\alpha_{k}$ and $S_{k}$ is not recursive in $T_{k}^{\left(\alpha_{k}\right)}$, then $\left\{D^{(\beta)}: D \in \mathscr{C}\right\}$ has no element of least degree.

Proof. Taking the proof of Lemma 1.3 and relativizing, we get a proof that for any sets $A, B$, and $W$, if $B \leq_{T}(W \oplus D)^{(\beta)}$ for all $D$ such that $A \leq_{T}$ $(W \oplus D)^{(\beta+1)}$, then $B \leq_{T} W^{(\beta)}$. Let $T_{k}^{*}=\bigoplus_{n \geq k} S_{n}$, and let $\mathscr{E}=\left\{D: T_{k}^{*} \leq_{T}\right.$ $\left.\left(T_{k} \oplus D\right)^{(\beta+1)}\right\}$. If $B \leq_{T}\left(T_{k} \oplus D\right)^{(\beta)}$ for all $D \in \mathscr{E}$, then $B \leq_{T} T_{k}^{(\beta)}$. Note that if $D \in \mathscr{E}$, then $T_{k} \oplus D \in \mathscr{C}$. Suppose $B \leq_{T} D^{(\beta)}$ for all $D \in \mathscr{C}$. Then $B \leq_{T}\left(T_{k} \oplus D\right)^{(\beta)}$ for all $D \in \mathscr{E}$, so $B \leq_{T} T_{k}^{(\beta)}$.

Now, suppose that $S_{k}$ is not recursive in $T_{k}^{\left(\alpha_{k}\right)}$. We must show that the set $\left\{D^{(\beta)}: D \in \mathscr{C}\right\}$ has no element of least degree. Hoping for a contradiction, suppose that there is an element $D_{0}^{(\beta)}$ of least degree, where $D_{0} \in \mathscr{C}$. We have $S_{k} \leq_{T} D_{0}^{\left(\alpha_{k}\right)}$. Since $D_{0}^{(\beta)} \leq_{T} D^{(\beta)}$ for all $D \in \mathscr{C}$, we get $D_{0}^{(\beta)} \leq_{T}$ $\left(T_{k}\right)^{(\beta)}$. Then since $\beta<\alpha_{k}, D_{0}^{\left(\alpha_{k}\right)} \leq_{T}\left(T_{k}\right)^{\left(\alpha_{k}\right)}$. Therefore, $S_{k} \leq_{T} T_{k}^{\left(\alpha_{k}\right)}$, a contradiction. This completes the proof. 


\section{Finite JUMPS}

For any structure $\mathbf{A}$, let $\operatorname{Set}(\mathbf{A})=\left\{D \subseteq \omega: \exists \mathbf{B}\left(\mathbf{B} \cong \mathbf{A} \& D(\mathbf{B}) \leq_{T} \quad D\right)\right\}$. If $\mathbf{A}$ is nontrivial, so that $\operatorname{Dg}(\mathbf{A})$ is closed upwards, then $\operatorname{Set}(\mathbf{A})=\{D \subseteq \omega$ : $\left.\exists \mathbf{B}\left(\mathbf{B} \cong \mathbf{A} \& D(\mathbf{B}) \equiv_{T} D\right)\right\}$, and we have $\operatorname{Dg}(\mathbf{A})=\{\operatorname{deg}(D): D \in \operatorname{Set}(\mathbf{A})\}$. This is true for orderings. In the present section, we shall obtain examples of orderings $\mathbf{A}_{n}$, for $2 \leq n<\omega$, such that $\mathbf{A}_{n}$ has $n$th jump degree but does not have $k$ th jump degree for $k<n$. We begin by describing a basic type of ordering that can be used to encode a set $S \subseteq \omega$. There is more than one possibility. In [L], Lerman considered the orderings $\tau(S)$ of form $a_{0}+\omega^{*}+\omega+a_{1}+\omega+\omega^{*}+$ $\cdots$, where $\left(a_{n}\right)_{n \in \omega}$ is a list of the elements of $S$ in order of magnitude. A slight modification of the proof of Lerman's theorem would yield the fact that $\operatorname{Dg}(\tau(S))=\left\{\operatorname{deg}(D): S\right.$ is r.e. in $\left.D^{\prime \prime}\right\}$.

Here we give a different ordering that encodes $S$. Let $\sigma^{*}(S)$ denote the "shuffle sum" composed of densely many copies of $\omega$ and of $n+1$ for $n \in S$. We show that $\operatorname{Dg}\left(\sigma^{*}(S)\right)$ is the same as $\operatorname{Dg}(\tau(S))$. We use Harrington's method of "workers" $[\mathrm{H}]$ in this result (Lemma 2.1) and also in the other main lemma of the section (Lemma 2.2). In each of these proofs, we need only three workers, and an ordinary infinite injury priority argument could be substituted (Soare produced such arguments in conversation with the third author). In some later lemmas, we use transfinitely many workers. In these later lemmas also, we could have avoided using workers, substituting the first author's " $\alpha$-systems" (see [A and $\mathrm{AK}]$ ). The reader interested in seeing further arguments using workers could consult [Mar, $\mathrm{K}_{1}, \mathrm{~K}_{3}$, or $\mathrm{K}_{4}$ ].

Lemma 2.1. For any $S \subseteq \omega, \operatorname{Dg}\left(\sigma^{*}(S)\right)=\left\{\operatorname{deg}(D): S\right.$ is r.e. in $\left.D^{\prime \prime}\right\}$.

Before proving Lemma 2.1, we indicate how it can be used. First, we show that for any degree $\mathbf{d}>\mathbf{0}^{\prime \prime}$, there is an ordering $\mathbf{A}_{2}$ such that $\mathbf{A}_{2}$ has 2 nd jump degree d but does not have 1 st jump degree. Let $S$ have degree d, and choose $D_{0}$ such that $D_{0}^{\prime \prime} \equiv_{T} S$. This is possible by Lemma 1.1. By Lemma 1.3 , if $\mathscr{C}=\left\{D: S \leq_{T} D^{\prime \prime}\right\}$, then $\left\{D^{\prime}: D \in \mathscr{C}\right\}$ has no element of least degree. Clearly, $D_{0} \in \mathscr{C}$ and $S$ is a set of least degree in $\left\{D^{\prime \prime}: D \in \mathscr{C}\right\}$. Let $\mathbf{A}_{2} \cong \sigma^{*}(S \oplus(\omega-S))$. By Lemma 2.1, $\operatorname{Dg}\left(\mathbf{A}_{2}\right)=\{\operatorname{deg}(D): S \oplus(\omega-S)$ is r.e. in $\left.D^{\prime \prime}\right\}=\{\operatorname{deg}(D): D \in \mathscr{C}\}$. Therefore, $\mathbf{A}_{2}$ has 2 nd jump degree $\mathbf{d}$ but does not have 1 st jump degree.

We can also produce an ordering $\mathbf{A}_{3}$ with 3 rd jump degree and not 2 nd, and we can make the 3 rd jump degree equal any $\mathbf{d}>\mathbf{0}^{(3)}$. To accomplish this, we choose $S$ such that $S$ is 3-generic, $S^{(3)}$ has degree d, and $S^{(3)} \equiv{ }_{T} 0^{(3)} \oplus S$. (This is possible by Lemma 1.1.) By Lemma 1.2 , if $\mathscr{C}=\left\{D: S\right.$ is r.e. in $\left.D^{\prime \prime}\right\}$, then $\left\{D^{\prime \prime}: D \in \mathscr{C}\right\}$ has no element of least degree. Let $\mathbf{A}_{3} \cong \sigma^{*}(S)$. We have $S \leq_{T} D^{(3)}$ for all $D \in \mathscr{C}$. Since $S^{(3)} \equiv_{T} 0^{(3)} \oplus S$, it follows that $S^{(3)} \leq_{T} D^{(3)}$ for all $D \in \mathscr{C}$. By Lemma 2.1, $\operatorname{Dg}\left(\mathbf{A}_{3}\right)=\{\operatorname{deg}(D): D \in \mathscr{C}\}$. Then $\mathbf{A}_{3}$ has 3rd jump degree $\mathbf{d}=\operatorname{deg}\left(S^{(3)}\right)$, and $\mathbf{A}_{3}$ does not have 2 nd jump degree. 
Proof of Lemma 2.1. We must show that $\operatorname{Set}(\mathbf{A})=\left\{D: S\right.$ is r.e. in $\left.D^{\prime \prime}\right\}$; i.e. $S$ is r.e. in $D$ iff there exists $\mathbf{A} \cong \sigma^{*}(S)$ such that $D(\mathbf{A}) \leq_{T} D$. One direction is easy. Suppose $\mathbf{A} \cong \sigma^{*}(S)$. Then $n \in S$ iff $\mathbf{A}$ has a maximal discrete set of size $n+1$, so $S$ is r.e. in $D(\mathbf{A})^{\prime \prime}$. The other direction is somewhat harder. Let $S$ be r.e. in $D^{\prime \prime}$. To produce $\mathbf{A} \cong \sigma^{*}(S)$ such that $D(\mathbf{A}) \leq_{T} D$, we use three workers. Workers 0,1 , and 2 have oracles for $D, D^{\prime}$, and $D^{\prime \prime}$, respectively. Let $f$ be a function recursive in $D^{\prime \prime}$ such that $\operatorname{ran}(f)=S$.

At stage $s$, Worker 2 computes $f(s)$. This is his only job. At stage $s$, Worker 1 guesses the value of $f(k)$ for each $k<s$ and produces an instruction $i_{s}$ for Worker 0 to follow. The instruction $i_{s}$ is actually based on $f(k)$ for a single $k=k_{s}$, where $k_{0}=0$ and for $s>0, k_{s}$ is the first $k$ such that the stage $s$ guess at $f(k)$ differs from the previous guess, or $k=k_{s}$ and Worker 1 has not finished work begun at stage $s-1$, or $k=k_{s-1}+1$. An essential feature of Worker 1's instructions is that it is "safe" for Worker 0 to carry out all of them. If Worker 1 changes his mind about the value of $f(k)$, any instruction to Worker 0 based on the old guess still stands. New instructions are issued that have a correcting effect. Suppose, for example, that at stage 1, Worker 1 guesses $f(0)=3$. Instruction $i_{1}$ says to build a discrete interval of size 4 . If at stage 2, Worker 1 guesses $f(0)=2$, then instruction $i_{2}$ says to make this discrete interval into an initial segment of a copy of $\omega$. Supposing that at stage 3 , Worker 1 again guesses $f(0)=2$, then instruction $i_{3}$ says to build a discrete interval of size 3 . If at stage 4 , Worker 1 guesses $f(0)=2$ and $f(1)=3$, then $i_{4}$ says to build a new discrete interval of size 4 .

Worker 0 guesses the instructions $i_{s}$ only and does not try to compute the function $f$. At stage $s$, Worker 0 has determined once and for all the ordering of a finite set of constants (the first few). In addition, Worker 0 has tentatively grouped various sets of constants, assigning them to be discrete sets of size $n+1$ for certain $n$ or initial segments of sets of type $\omega$. At stage $s$, Worker 0 guesses $i_{k}$ for $k<s$. Worker 0 bases his work on $i_{k}$ for a particular $k$; namely, the first $k$ such that either the current guess at $i_{k}$ differs from the previous one or else the work at stage $s-1$ was based on $i_{k-1}$.

First, suppose that at stage $s$, Worker 0 is trying to carry out $i_{k}$, where at stage $s-1$, the task was $i_{k-1}$. If $i_{k}$ says to build a discrete set of some finite size, then Worker 0 does that. In addition, Worker 0 works toward density, putting into the intervals between the various discrete sets, and before the first and after the last, further discrete sets of all types presently represented. Moreover, if two different instructions ( $i_{5}$ and $i_{6}$, for example) call for sets of the same finite size (say 3), and no later instruction makes these into copies of $\omega$, then when Worker 0 is working toward density, he adds sets (of size 3 ) on behalf of each instruction. To complete the work at stage $s$, Worker 0 adds an element at the end of each finite set that is supposed to become a copy of $\omega$.

Suppose that instruction $i_{k}$ says to convert into a copy of $\omega$ the finite set created in response to some earlier instruction $i_{j}$ for $j<k$. Then Worker 0 
makes the appropriate change in assignment for the finite set originally created in carrying out $i_{j}$ and also for any copies of the set that have been produced in work toward density. This does not mean that all finite sets of a particular size are converted into copies of $\omega$. It may be that instructions $i_{5}$ and $i_{6}$ both call for building sets of size 3 , and only those resulting from $i_{6}$ are being converted into copies of $\omega$.

Suppose that at stage $s$ Worker 0 has chosen to work on instruction $i_{k}$ because his current guess at $i_{k}$ differs from the previous guess. Worker 0 must correct mistakes based on the old guess without changing the ordering of the constants. Suppose that $i_{k}$ seemed at first to ask for a set of size 5, and by stage $s$, Worker 0 has built several sets of size 5 in connection with $i_{k}$. If $i_{k}$ now seems to ask for sets of size 6 , Worker 0 converts the sets of size 5 into initial segments of copies of $\omega$ and starts new sets of size 6. Suppose that Worker 0's previous guess at $i_{k}$ led him to convert sets of size 3 , created in response to $i_{j}$ for some $j<k$, into copies of $\omega$. These sets will have acquired new elements at the end. Suppose that Worker 0 now believes that $i_{k}$ is asking for something else (a set of size 15, say). He designates the old sets of size 3 (created for $i_{j}$ ) as sets of size 3 again and converts the segments added at the end into copies of $\omega$.

It is clear that if $n \in S$, then Worker 1 eventually asks for a discrete set of size $n+1$, and Worker 0 eventually cooperates so that densely many discrete sets of size $n+1$ are produced. Worker 0 may believe infinitely often that he is being asked to convert these into copies of $\omega$, because of wrong guesses at infinitely many different $i_{k}$ 's. However, each wrong guess is eventually discovered, and the work toward density between errors has the effect of making the sets of size $n+1$ maximal discrete in the finished model. If $n \notin S$, then Worker 1 may wrongly guess $f(k)=n$ for infinitely many $k$. He will in that case issue infinitely many instructions calling for sets of size $n+1$. However, each such instruction is followed (when Worker 1 discovers that $f(k) \neq n$ ) by an instruction to convert the appropriate family of sets of size $n+1$ into copies of $\omega$. Therefore, the construction results in a copy $\mathbf{A}$ of $\sigma^{*}(S)$ such that $D(\mathbf{A}) \leq_{T} D$. This completes the proof of the lemma.

Note that Lemma 2.1 is true uniformly. That is, we have a uniform procedure for enumerating $S$ using an oracle for $D(\mathbf{B})^{\prime \prime}$, for any $S$ and any $\mathbf{B} \cong \sigma^{*}(S)$. Also, given a procedure for enumerating $S$ using an oracle for $D^{\prime \prime}$, we can find a procedure for computing $\chi_{D(\mathbf{B})}$ for some $\mathbf{B} \cong \sigma^{*}(S)$ using an oracle for $D$. (The new procedure depends on the old procedure, not on $D$ and $S$.)

The next lemma characterizes $\operatorname{Dg}(Z \cdot \mathbf{A})$ in terms of $\operatorname{Dg}(\mathbf{A})$.

Lemma 2.2. For any ordering $\mathbf{A}$ and any degree $\mathbf{d}, \mathbf{d} \in \operatorname{Dg}(Z \cdot \mathbf{A})$ iff $\mathbf{d}^{\prime \prime} \in$ $\operatorname{Dg}(\mathbf{A})$.

Proof. It is enough to show that for any $D \subseteq \omega$, the following are equivalent:

(1) there exists $\mathbf{B} \cong Z \cdot \mathbf{A}$ such that $D(\mathbf{B}) \leq_{T} D$,

(2) there exists $\mathbf{C} \cong \mathbf{A}$ such that $D(\mathbf{C}) \leq_{T} D^{\prime \prime}$. 
To see why (1) implies (2), note that if $\mathbf{B} \cong Z \cdot \mathbf{A}$, then the relation $\sim_{0}$ on $\mathbf{B}$ is recursive in $D(\mathbf{B})^{\prime \prime}$, and $\mathbf{A} \cong \mathbf{B} / \sim_{0}$. We must show that (2) implies (1). Let $\mathbf{C} \cong \mathbf{A}$, where $D(\mathbf{C}) \leq_{T} D^{\prime \prime}$. We must construct $\mathbf{B} \cong Z \cdot \mathbf{A}$ such that $D(\mathbf{B}) \leq_{T} D$. As in the previous lemma, we use three workers. Worker 0 has an oracle for $D$, Worker 1 has an oracle for $D^{\prime}$, and Worker 2 has an oracle for $D^{\prime \prime}$. Although the universe of $\mathbf{C}$ is $\omega$, and $\mathbf{B}$ will also have universe $\omega$, we write $c_{i}$ for the $i$ th element of $\mathbf{C}$ and think of $\mathbf{B}$ as made up of constants $b_{i}$. At stage $s$, Worker 2 computes the ordering of $c_{k}$ for $k \leq s$. He writes this down as $o_{s}$.

At stage $s$, Worker 1 guesses $o_{k}$ for $k \leq s$. Let $k_{s}$ be the first $k$ such that either the current guess at $o_{k}$ differs from the preceding guess or else $k=k_{s-1}+1$. Worker 1 acts on $o_{k}$. What Worker 1 produces at stage $s$ is an instruction $i_{s}$ for Worker 0 . Each instruction either asks Worker 0 to start a new $Z$-piece in a certain interval (between two $Z$-pieces requested in an earlier instruction, or at the beginning or end), or else says to destroy a $Z$-piece requested earlier. Instruction $i_{0}$ will ask for a $Z$-piece $Z_{0}$ (which Worker 1 thinks of as corresponding to $c_{0}$ ). Suppose at stage 1 , Worker 1 guesses $c_{0}<c_{1}$. Then instruction $i_{1}$ will ask for a new $Z$-piece $Z_{1}$ to the right of $Z_{0}$. (Worker 1 associates $Z_{1}$ with $c_{1}$.) Suppose at stage 4 , Worker 1 guesses $c_{1}<c_{0}$. Then $i_{2}$ says to destroy $Z_{2}$, and $i_{3}$ says to create a new $Z$-piece (say $Z_{3}$ ) to the left of $Z_{1}$. The full sequence of instructions $\left(i_{s}\right)_{s \in \omega}$ has the feature that if Worker 0 follows the instructions and also fleshes out with constants any $Z$-piece that is not destroyed, the result is a copy of $Z \cdot C$.

We must show that Worker 0 is able to carry out the instructions, adjusting for his own mistakes. At stage $s$, Worker 0 has decided once and for all on an ordering for finitely many of the constants $b_{i}$ (the first few). In addition, Worker 0 has tentatively grouped the constants into nonoverlapping $Z$-pieces. At stage $s$, Worker 0 guesses instructions $i_{k}$ for $k \leq s$. At stage 0 , Worker 0 acts on $i_{0}$, and at stage $s>0$, Worker 0 acts on $i_{k}$ for the first $k$ such that either the current guess at $i_{k}$ differs from the previous guess or else the instruction acted on last was $i_{k-1}$.

Suppose that instruction $i_{k}$ asks for a new $Z$-piece in some interval, say between $Z_{6}$ and $Z_{2}$ (where these were mentioned in previous instructions). Worker 0 starts a new $Z$-piece in the right interval and puts into it the first unused constant. Worker 0 will already have finite groups of constants assigned to $Z_{6}$ and to $Z_{2}$, and there may be other groups of constants between these (resulting from wrong guesses that Worker 0 has not yet discovered). Worker 0 will avoid putting the new constant between elements of a group. Suppose that instead of asking for a new $Z$-piece, instruction $i_{k}$ calls for destroying an existing $Z$-piece. Thisi $Z$-piece may have acquired several constants. Now, Worker 0 puts these constants into the nearest $Z$-piece on the left (if there is one) or on the right. After carrying out instruction $i_{k}$, Worker 0 adds an element to the beginning and end of all existing $Z$-pieces. 
We must say how Worker 0 corrects his errors. It may be that Worker 0 created a $Z$-piece in response to an earlier guess at $i_{k}$ that now seems wrong. To correct, Worker 0 attaches the constants presently in this $Z$-piece to an adjacent one. Making the other possible kind of error. Worker 0 may have destroyed a $Z$-piece in response to a guess at $i_{k}$ that now seems wrong. Suppose that in responding to $i_{k}$, Worker 0 destroyed the $Z$-piece $Z_{10}$, attaching its constants to an adjacent $Z$-piece $Z_{3}$ on the left. To correct now, Worker 0 separates $Z_{10}$ from $Z_{3}$, apportioning the constants in the obvious way. After making a correction, Worker 0 acts on the new guess at $i_{k}$, and then adds elements to all of the existing $Z$-pieces as above.

All of the $Z$-pieces that are started incorrectly are eventually destroyed. The $Z$-pieces that ought to be distinct are separated each time they become joined, and while they are separated (between errors), new elements are inserted to make them grow apart. For a given element $c_{i}$ of $\mathbf{C}$, Worker 1 may use several names $Z_{j}$ for the $Z$-piece corresponding to $c_{i}$. As Worker 1 discovers mistakes in his guesses at the place for $c_{i}$ in $\mathbf{C}$, he calls for $Z_{j}$ 's to be destroyed, and the name that sticks is the one attached after the last mistake. The elements of a $Z$-piece are chosen by Worker 0 . There is one put in to start the $Z$-piece; finite collections of elements enter a $Z$-piece when adjacent $Z$-pieces are destroyed, and other elements are added when the $Z$-piece is routinely enlarged at the end of a stage. This completes the proof of Lemma 2.2.

Lemma 2.2 is true uniformly in the same way that Lemma 2.1 is. Combining Lemmas 2.1 and 2.2, we get the following result.

Lemma 2.3. For any $S \subseteq \omega$ and any $n \in \omega$,

(a) $\operatorname{Dg}\left(Z^{n} \cdot \sigma^{*}(S)\right)=\left\{\operatorname{deg}(D): S\right.$ is r.e. in $\left.D^{(2 n+2)}\right\}$, and

(b) $\operatorname{Dg}\left(Z^{n} \cdot \sigma^{*}(S \oplus(\omega-S))\right)=\left\{\operatorname{deg}(D): S \leq_{T} D^{(2 n+2)}\right\}$.

Now, we are in a position to prove a general theorem on finite jumps.

Theorem 2.4. For each $n$ such that $2 \leq n<\omega$, there is an ordering $\mathbf{A}_{n}$ with $n$th jump degree and not $k$ th for $k<n$. Moreover, if $\mathbf{d}>\mathbf{0}^{(n)}$, then we can make $\mathbf{d}$ the nth jump degree of $\mathbf{A}_{n}$.

Proof. For $n=2+2 m$, choose $S$ of degree d and let $D_{0}$ be $n$-generic, such that $D_{0}^{(n)} \equiv_{T} S$. This is possible by Lemma 1.1. Let $\mathbf{A}_{n} \cong Z^{m} \cdot \sigma^{*}(S \oplus(\omega-S))$. By Lemma $2.3(\mathrm{~b})$, if $\mathscr{C}$ is the set $\left\{D: S \leq_{T} D^{(n)}\right\}$, then $\operatorname{Dg}\left(\mathbf{A}_{n}\right)=\{\operatorname{deg}(D): D \in$ $\mathscr{C}\}$. Clearly, $S$ is an element of least degree in $\left\{D^{(n)}: D \in \mathscr{C}\right\}$. By Lemma 1.3, $\left\{D^{(n-1): D \in f}\right\}$ has no element of least degree. Therefore, $\mathbf{A}_{n}$ has $n$th jump degree $\mathbf{d}=\operatorname{deg}(S)$, and $\mathbf{A}_{n}$ does not have $n-1$ st jump degree. For $n=3+2 m$, choose $S$ to be $n$-generic such that $S^{(n)}$ has degree d. Then $S^{(n)} \equiv_{T} 0^{(n)} \oplus S$. This is possible by Lemma 1.1. By Lemma 1.2 , if $\mathscr{C}=\left\{D: S\right.$ is r.e. in $\left.D^{(n-1)}\right\}$, then $\left\{D^{(n-1)}: D \in \mathscr{C}\right\}$ has no element of least degree. Let $\mathbf{A}_{n} \cong Z^{m} \cdot \sigma^{*}(S)$. By Lemma 2.3(a), $\operatorname{Dg}\left(\mathbf{A}_{n}\right)=\{\operatorname{deg}(D): D \in \mathscr{C}\}$. Clearly, $S \in \mathscr{C}$, and since $S^{(n)} \equiv_{T} 0^{(n)} \oplus S, S^{(n)}$ is an element of least degree in $\left\{\operatorname{deg}(D)^{(n)}: D \in \mathscr{C}\right\}$. It 
follows that $\mathbf{A}_{n}$ has $n$th jump degree $\mathbf{d}=\operatorname{deg}\left(S^{(n)}\right)$, and $\mathbf{A}_{n}$ does not have $n-1$ st jump degree. This completes the proof of Theorem 2.4.

\section{CONSTRUCTIONS FOR $\omega, \omega+1$ AND $\omega+2$}

This section is not essential to the logical development of our results on orderings with $\alpha$ th jump degree for arbitrary recursive ordinals $\alpha$. The next section includes general results for limit ordinals and for the first and second successors of limit ordinals, and these results certainly apply to $\omega, \omega+1$, and $\omega+2$. We shall not actually apply any of the results of the present section. Our aim here is to introduce in a simpler setting some of the ideas to be used in the next section. In particular, the proof of Lemma 3.2 is the first place we use a limit worker. We explain in some detail how this worker delegates his actions to the workers below. Lemma 3.2 will be generalized in the next section, where the proof involves infinitely many limit workers.

We begin by combining some orderings of the form $Z^{m} \cdot \sigma^{*}(S)$ to produce an ordering $\mathbf{A}_{\omega}^{*}$ that has $\omega$ th jump degree and does not have $n$th jump degree for any $n<\omega$. (The ordering that we call $\mathbf{A}_{\omega}$ in $\S 4$ will be slightly different.) For $S \subseteq \omega \times \omega$, let $S_{n}=\{k:(n, k) \in S\}$, and let $\rho(S)=\sum_{n<\omega} Z^{n} \cdot \sigma^{*}\left(S_{n}\right)$. The following lemma characterizes $\operatorname{Dg}(\rho(S))$.

Lemma 3.1. For any $S \subseteq \omega \times \omega, \operatorname{Dg}(\rho(S))=\left\{\operatorname{deg}(D): S_{n}\right.$ is r.e. in $D^{(2+2 n)}$ uniformly in $n\}$.

Before proving this lemma, we use it to produce $\mathbf{A}_{\omega}^{*}$. It follows from Lemma 1.1 that for any $\mathbf{d}>\mathbf{0}^{(\omega)}$, there is an $\omega$-generic set $S$ such that $S^{(\omega)} \equiv_{T} 0^{(\omega)} \oplus S$ and $S^{(\omega)}$ has degree d. We take $S \subseteq \omega \times \omega$. It follows from genericity that for all $m$ and $k, S_{k+1}$ is not recursive in $\left(S_{0} \oplus \cdots \oplus S_{k}\right)^{(m)}$. Let $\mathbf{A}_{\omega}^{*} \cong \rho(S)$. If $\mathscr{C}=\left\{D: S_{k}\right.$ is r.e. in $D^{(2+2 k)}$ uniformly in $\left.k\right\}$, then by Lemma 2.4, $\operatorname{Dg}\left(\mathbf{A}_{\omega}^{*}\right)=\{\operatorname{deg}(D): D \in \mathscr{C}\}$. Clearly, $S \in \mathscr{C}$, and if $D \in \mathscr{C}$, then $S^{(\omega)} \leq_{T} D^{(\omega)}$. Therefore, $\mathbf{A}_{\omega}^{*}$ has $\omega$ th jump degree d. To show that for $n \in \omega, \mathbf{A}_{\omega}^{*}$ does not have $n$th jump degree, suppose it did. This means that there is some $D_{0} \in \mathscr{C}$ such that $D_{0}^{(n)} \leq_{T} D^{(n)}$ for all $D \in \mathscr{C}$. In particular, $D_{0}^{(n)} \leq_{T} D^{(n)}$ for those $D$ in $\mathscr{C}$ such that $S_{k} \leq_{T} D^{(2+2 k)}$ uniformly in $k$. By Lemma 1.4 , if $2+2 k>n$, then $D_{0}^{(n)} \leq_{T}\left(S_{0} \oplus \cdots \oplus S_{k-1}\right)^{(2+2 k)}$. Then $D_{0}^{(2+2 k)} \leq_{T}\left(S_{0} \oplus \cdots \oplus S_{k-1}\right)^{(m)}$, for some $m$. Since $S_{k}$ is r.e. in $D_{0}^{(2+2 k)}$, we have $S_{k} \leq_{T} D_{0}^{(3+2 k)}$, so $S_{k} \leq_{T}\left(S_{0} \oplus \cdots \oplus S_{k-1}\right)^{(m+1)}$, a contradiction. Therefore, $\mathbf{A}_{\omega}^{*}$ does not have $n$th jump degree.

Proof of Lemma 3.1. It must be shown that $\operatorname{Set}(\rho(S))=\left\{D \subseteq \omega: S_{n}\right.$ is r.e. in $D^{(2+2 n)}$ uniformly in $\left.n\right\}$; i.e., for any $D$, the following are equivalent:

(1) there exists $\mathbf{B}$ such that $\mathbf{B} \cong \rho(S)$ and $D(\mathbf{B}) \leq_{T} D$,

(2) $S_{n}$ is r.e. in $D^{(2+2 n)}$ uniformly in $n$. 
To show that (1) implies (2), let $\mathbf{B} \cong \rho(S)$. The relation $\sim_{n}$ is recursive in $D(\mathbf{B})^{(2 n)}$ uniformly in $n$, and $S_{n}$ is r.e. in $D\left(\mathbf{B} / \sim_{n}\right)^{(2)}$ uniformly in $n$. It follows that if $D(\mathbf{B}) \leq_{T} D$, then $S_{n}$ is r.e. in $D^{(2+2 n)}$ uniformly in $n$. To show that (2) implies (1), we use the uniform versions of the lemmas from $\S 2$. If $S_{n}$ is r.e. in $D^{(2 n+2)}$ uniformly in $n$, then there is a sequence of orderings $\left(\mathbf{B}_{n}\right)_{n \in \omega}$ such that $\mathbf{B}_{n} \cong \sigma^{*}\left(S_{n}\right)$ and $D\left(\mathbf{B}_{n}\right) \leq_{T} D^{(2 n)}$ uniformly in $n$. Then there is a sequence of orderings $\mathbf{C}_{n} \cong Z^{n} \cdot \mathbf{B}_{n}$ with $D\left(\mathbf{C}_{n}\right) \leq_{T} D$ uniformly in $n$. Taking the sum of the $\mathbf{C}_{n}$ 's, we get the desired $\mathbf{B} \cong \rho(S)$ such that $D(\mathbf{B}) \leq_{T} D$. This completes the proof of Lemma 3.1.

To produce an ordering that has $\omega+1$ st jump degree but not $\omega$ th, or one that has $\omega+2$ nd jump degree but not $\omega+1$ st, we need a new method for encoding sets. For $S \subseteq \omega$, let $\nu^{\omega}(S)$ be the sum of densely many copies of $r+1+\sum_{1 \leq i \leq M} Z^{i}$ for all $r$ and $M$ such that $1 \leq r<\omega$ and $1<M<\omega$, and $r+1+\sum_{1 \leq i<\omega} Z^{i}$ for all $r \in S$. The next lemma characterizes $\operatorname{Dg}\left(\nu^{(\omega)}(S)\right)$. The proof involves a construction by infinitely many workers. One of these is a limit worker. In $\S 4$, when we generalize this lemma, we shall have to face thickets of limit workers. Our hope is that in reading the proof of Lemma 3.2, the reader will learn how the workers below the limit worker act as proxies for the limit worker. In $\S 4$, there will be added scheduling difficulties since one worker may act as a proxy for infinitely many different limit workers.

Lemma 3.2. For any $S \subseteq \omega, \operatorname{Dg}\left(\nu^{\omega}(S)\right)=\left\{\operatorname{deg}(D): S\right.$ is r.e. in $\left.D^{(\omega+1)}\right\}$.

Proof. We show that for any $D \subseteq \omega$, there exists $\mathbf{B} \cong \nu^{\omega}(S)$ such that $D(\mathbf{B}) \leq_{T}$ $D$ iff $S$ is r.e. in $D^{(\omega+1)}$. First, suppose that $\mathbf{B} \cong \nu^{\omega}(S)$. Then $r \in S$ iff $\mathbf{B}$ has an element $a$, located in a maximal discrete set of size $r+1$, with the property that for each $n \in \omega$, there is some $b$ such that $a \sim_{n+1} b$ and not $a \sim_{n} b$. From this, it is easy to see that $S$ is r.e. in $D(\mathbf{B})^{(\omega+1)}$. Now, suppose that $S$ is r.e. in $D^{(\omega+1)}$. Let $f$ be a function that enumerates $S$, with $f \leq_{T} D^{(\omega+1)}$. We must produce $\mathbf{B} \cong \nu^{\omega}(S)$ such that $D(\mathbf{B}) \leq_{T} D$.

The construction involves infinitely many workers. For each $\alpha \leq \omega+1$, Worker $\alpha$ has an oracle for $D^{(\alpha)}$. At stage $s$, Worker $\omega+1$ computes $f(s)$. At stage $s$, Worker $\omega$ can guess the value of $f(k)$ for various $k$. We describe the guessing process in some detail, since we want it also to be done by various workers below $\omega$. At stage $s$, Worker $\omega$ has an approximation of $D^{(\omega+1)}$ consisting of those $e$ such that for some $C \leq s, C$ is a halting computation of $\varphi_{e}^{D^{(\omega)}}(e)$ in which there are no questions asked about $D^{(n)}$ for $n>s$. Let $D_{s}$ be this approximation. Note that if $e \in D^{(\omega+1)}$, then $e \in D_{s}$ for all sufficiently large $s$, and if $e \notin D^{(\omega+1)}$, then $e \notin D_{s}$ for any $s$. At stage $s$, Worker $\omega$ can try to compute $f(k)$, using $D_{s}$ instead of $D^{(\omega+1)}$ and bounding the computations by $s$. Let $f^{*}(k, s)$ represent the stage $s$ guess at $f(k)$ obtained this way, if it exists. By assigning garbage values if necessary, we make $f^{*}$ 
total. The important features of $f^{*}$ are (1) for each $j$, there is some $s_{0}$ such that for all $k \leq j$ and all $s \geq s_{0}, f^{*}(k, s)=f(k)$, and (2) the functions $f_{n}(k)=f^{*}(k, n)$ are uniformly recursive in $D^{(n)}$. Note that for each $n$, Worker $n$ can compute $f_{n}$ just as Worker $\omega$ does.

The model B will consist of densely many "finite chains" of the form $r+$ $1+\sum_{1 \leq i \leq M} Z^{i}$ for $1 \leq r<\omega$ and $1<M<\omega$, and "infinite chains" of the form $r+1+\sum_{1 \leq i<\omega} Z^{i}$ for $r \in S$. We shall start the construction with some constants already arranged in chains. Initially, there are only finite chains. In the course of the construction, the workers will convert some of these into infinite chains. Each chain is assigned a "type". Identical-looking chains, of form $r+1+\sum_{1 \leq i \leq M} Z^{i}$ initially, may be assigned different types. The chain may be either not extendible, in which case we write $(r, M)$-NE for the type, or possibly extendible if $f(k)=r$, for some particular $k$, in which case we write $(r, M)$-E $\mid f(k)$ for the type. Let $\left(\tau_{i}\right)_{i \in \omega}$ be a list of the types, with all possible parameters $r, M$, and possibly $k$.

We partition the rationals into infinitely many dense subsets $\left(Q_{i}\right)_{i \in \omega}$. To each rational $q$, we assign a type of chain, such that the type assigned to $q$ is $\tau_{i}$ if $q \in Q_{i}$. We also assign to each rational $q$ an infinite set of constants $B_{q}$. The sets $B_{q}$ are disjoint, and the universe of $\mathbf{B}$ will be $\bigcup\left\{B_{q}: q \in Q\right\}$. If $q<r$, then in the ordering of $\mathbf{B}$, the elements of $B_{q}$ will precede the elements of $B_{r}$. If $q$ is assigned the type $(r, M)$-NE, then $B_{q}$ will be arranged in a particular predetermined way to form a copy of $r+1+\sum_{1 \leq i \leq M} Z^{i}$. Suppose that $q$ is assigned the type $(r, M)$-E $\mid f(k)$. The constants from $B_{q}$ will become a copy of $r+1+\sum_{1 \leq i<\omega} Z^{i}$ if $M$ is sufficiently large and $f(k)$ actually is $r$. Otherwise, $B_{q}$ will form a copy of $r+1+\sum_{1 \leq i \leq K} Z^{i}$ for some $K \geq M$. A predetermined subset of $B_{q}$ will be arranged in a predetermined way to form the initial segment $r+1+\sum_{1 \leq i \leq M-1} Z^{i}$, and there will be infinitely many constants from $B_{q}$ left for constructing the terminal segment $\sum_{M \leq i<\omega} Z^{i}$, or $\sum_{M \leq i \leq K} Z^{i}$.

Everything so far is done recursively. It is the construction of the terminal segments that uses the oracle $D$ and involves the workers. Clearly, the different terminal segments (associated with different $q$ 's) can be constructed independently, so we look at just one. Suppose that the chain we are considering is initially assigned the type $(r, M)$-E $\mid f(k)$. Let $B^{*}$ be the set of unused constants from the appropriate $B_{q}$. The terminal part of the chain-the part being constructed-will start with a copy of $Z^{M}$, and this may be followed by copies of $Z^{i}$ for some $i>M$. Let $T_{M}$ be the task of constructing a copy of $Z^{M}$, and let $T_{i}$, for $i>M$, be the task of deciding whether to construct a copy of $Z^{i}$ and then constructing it, if that is appropriate. For $i>0$, the copy of $Z^{i}$ is made up of copies of $Z^{i-1}$, which are made up of copies of $Z^{i-2}$, etc., and constructing the copy will require cooperation of several workers. 
At stage $s$, Worker $n$ has a picture of the terminal segment of the chain. The picture consists of a finite (possibly empty) ordered set of copies of $Z^{n+1}$, which we shall call $n$-blocks. If $n>0$, then Worker $n$ 's picture also has within each $n$-block a finite ordered set of $(n-1)$-blocks. Worker 0's picture consists of finitely many 0 -blocks (copies of $Z$ ), with a finite ordered set of constants in each. What Worker $n$ does first at stage $s$ is to revise his picture. In addition, for $n>0$, Worker $n$ issues an instruction $i_{n, s}$ for Worker $n-1$. Worker 0 does not issue instructions after revising his picture. Instead, he extends his ordering to the next few constants from $B^{*}$.

At stage 0 , Worker $M-1$ initiates Task $T_{M}$ by putting the first $(M-1)$ block into his picture (to the right of the predetermined part of the chain). If $M>1$, then Worker $M-1$ also puts an $(M-2)$-block inside the $(M-1)$ block and issues an instruction $i_{M-1,0}$ telling Worker $M-2$ to produce an $(M-2)$-block (also to the right of the predetermined part of the chain). If $M=1$, then Worker 0 initiates Task $T_{1}$ by putting a 0 -block into the picture (on the right), and then putting the first constant into it. For $n \geq M$, Worker $n$ computes $f^{*}(k, n)$ at stage 0 . If $f^{*}(k, n)=r$ and Worker $n-1$ has initiated Task $T_{n}$ at stage 0 , then Worker $n$ initiates Task $T_{n+1}$ at stage 0 by putting an $n$-block into his picture (on the right). If Worker $n$ has initiated Task $T_{n+1}$ at stage 0 , then in $i_{n, 0}$, he tells Worker $n-1$ to put an $(n-1)$-block at the end (after the $n$-block that was created in initiating Task $T_{n}$ ). If Worker $n$ did not initiate Task $T_{n+1}$ at stage 0 and $n>0$, then $i_{n, 0}$ says to do nothing.

At stage $s>0$, Worker $n$ first guesses $i_{n+1, k}$ for $k<s$. Let $k_{s}$ be the least $k$ such that either the current guess at $i_{n+1, k}$ differs from the previous guess or else $k=k_{s-1}+1$. Worker $n$ revises his picture on the basis of $i_{n+1, k}$ for $k=k_{s}$. Suppose first that $k_{s}=k_{s-1}+1$. Then Worker $n$ is trying to carry out the instruction just after the one he did at the previous stage. Unless the instruction is trivial, it says to produce a new $n$-block in a location described in terms of preceding instructions (e.g., just before the $n$-block created in response to $\left.i_{n+1,3}\right)$. Worker $n$ can carry out the instruction immediately, locating a new block properly in his picture.

Now, suppose that the current guess at the instruction differs from the previous one. Worker $n$ may already have carried out his previous guess at the instruction, at some stage $t<s$. Suppose that at stage $t$ Worker $n$ believed the instruction said to start an $n$-block in a certain interval and now he believes that the instruction says something else. Worker $n$ revises his picture so that none of the $n$-blocks created at stage $t$ or after exist. Between stage $t$ and stage $s$, Worker $n$ may have issued instructions asking Worker $n-1$ to produce $(n-1)$-blocks to start fleshing out these $n$-blocks. Now that the $n$-blocks no longer exist, Worker $n$ associates each of the $(n-1)$-blocks with the nearest existing $n$-block on the left (if there is one) or on the right. Worker $n-1$ never thought about the $n$-blocks anyway, so he does not have to adjust for Worker $n$ 's mistake. After collapsing the unwanted $n$-blocks and stowing the associated 
$(n-1)$-blocks in this way, Worker $n$ carries out his current guess at the chosen instruction.

We have said how at stage $s$ Worker $n$ revises his picture. If $n>0$, we must also say how Worker $n$ determines $i_{n, s}$. To each $s$, we assign a triple $(t, j, k)$. We think of the $n$-blocks as having names $B_{t}$ for certain $t \in \omega$. (This simply means that the blocks are assigned numbers.) If the picture at stage $s$ contains an $n$-block $B_{t}$ (assigned number $t$ ), and for the triple $(t, j, k)$ assigned to stage $s$, we have $j=0$ (or $j=1$ ), then $i_{n, s}$ tells Worker $n-1$ to construct an $(n-1)$-block located at the beginning (or end) of $B_{t}$. In the instruction, the place is described in terms of previous instructions and does not mention $B_{t}$ (for example, the place may be "just after the block created in response to instruction $i_{n, 7}$ "). If the number $s$ does not code a triple of the type described, then $i_{n, s}$ says to do nothing. At stage $s$, after Worker 0 has revised his picture of the 0 -blocks, he puts a new element at the beginning and end of each 0 -block in his current picture.

Claim. For all $n>0$, all $s$, Worker $n-1$ eventually carries out instruction $i_{n, s}$ in a way that is permanent.

Eventually, Worker $n-1$ has a correct and stable guess at $i_{n, k}$ for all $k \leq s$. If Worker $n-1$ has carried out $i_{n, k}$ for all $k<s$, then he will carry out $i_{n, s}$.

Claim. If an instruction $i_{n, s}$ calls for creating an $(n-1)$-block, then the block is fleshed out in the model $\mathbf{B}$.

For $n=1$, Worker 0 puts constants into the ordering to form the 0 -blocks in B matching his picture. For $n>1$, Worker $n-1$ issues instructions asking Worker $n-2$ to create $(n-2)$-blocks to flesh out the $(n-1)$-blocks.

Claim. All tasks $T_{n}$ are carried out.

The first task, namely $T_{M}$, is carried out since Worker $M-1$ initiates the task at stage 0 , and among the instructions $i_{M-1, s}$ are infinitely many calling for $(M-2)$-blocks to flesh out the desired $(M-1)$-block. Suppose that $T_{n}$ is initiated at stage 0 , and $T_{n}$ is eventually carried out with cooperation from below. If $f^{*}(k, n+1)=r$, then Worker $n$ initiates Task $T_{n+1}$ at stage 0 . Worker $n$ will, at various stages $s$, issue instructions $i_{n, s}$ that call for $(n-1)$-blocks to flesh out the desired $n$-block. Therefore, Task $T_{n+1}$ is also carried out (with cooperation). It follows that if $f(k)=r$ and for all $n \geq M$, $f^{*}(k, n)=r$, then the chain becomes a copy of $r+1+\sum_{1 \leq i<\omega} Z^{i}$. If there is some first $K \geq M$ such that $f^{*}(k, K) \neq r$, then the chain becomes a copy of $r+1+\sum_{1 \leq i \leq K} Z^{i}$. (Worker $K$ does not initiate the task $T_{K+1}$.)

We could describe a total output for Worker $n$ at stage $s, o(n, s)$. If we made this precise, we would have a recursive function $\sigma$ with $\sigma(n, s)$ depending on $\sigma(n, k)$ and $\sigma(n+1, k)$ for $k<s$, such that $\varphi_{\sigma(n, s)}^{D^{(n)}}(n, s)=o(n, s)$. For $n=0, \varphi_{\sigma(0, s)}^{D}(0, s)=\varphi_{e}^{D}(s)$ yields an enumeration of the diagram of an ordering $\mathbf{B} \cong \nu^{\omega}(S)$ such that $D(\mathbf{B})$ is recursive in $D$. 


\section{CONSTRUCTIONS FOR ARBITRARY RECURSIVE ORDINALS}

We begin this section by describing a method for encoding an arbitrary set $S$ in an ordering $\mathbf{A}_{\alpha}(S)$ for each recursive ordinal $\alpha$. In fact, we assign to each ordinal notation $a \in O$ an ordering $\mathbf{A}_{c \gamma}(S)$ that encodes $S$. What we are actually describing is a recursive function that takes the ordinal notation $a$ to an index $e$ such that for all sets $S, \varphi_{e}^{S}$ is the characteristic function of $D\left(\mathbf{A}_{a}(S)\right)$. For the most part, we ignore the distinction between an ordinal notation $a \in O$ and the ordinal $\alpha=|a|$, and we write $\alpha$ instead of $a$. We shall think of the set $S$ sometimes as a subset of $\omega \times \omega$ (if $\alpha$ is a limit ordinal) and sometimes as a subset of $\omega$ (if $\alpha$ is not a limit ordinal). When we are thinking of $S$ as a subset of $\omega \times \omega$, we write $S_{n}$ for $\{k:(n, k) \in S\}$ (just as we did in $\S 3$ ).

We need some definitions. In $\S 3$, we used an ordering which we called $\nu^{\omega}(S)$. Generalizing now to arbitrary recursive limit ordinals $\alpha$, let $\nu^{\alpha}(S)$ be a sum of densely many copies of $r+1+\sum_{1 \leq \xi<\beta} Z^{\beta}$ for $r \in \omega, 1 \leq \beta<\alpha$, and $r+1+\sum_{1 \leq \xi<r} Z^{\beta}$ for $r \in S$. Also, if $\left(\alpha_{n}\right)_{n \in \omega}$ is the fundamental sequence picked out by some notation for $\alpha$, let $\left(\alpha_{n}^{\prime}\right)_{n \in \omega}$ be the new sequence defined as follows:

(1) if $\alpha_{n}$ is finite, then $\alpha_{n}^{\prime}$ is the first positive even integer $k$ such that $k \geq 3, k \geq \alpha_{n}$, and if $n>0$, then $k>\alpha_{n-1}^{\prime}$,

(2) if $\alpha_{n}$ is infinite and $\beta$ is the greatest limit ordinal such that $\beta \leq \alpha_{n}$, then $\alpha_{n}^{\prime}$ is the first ordinal $\gamma$ such that $\gamma \geq \alpha_{n}, \gamma=\beta+k$ for some odd integer $k$, and if $n>0$, then $\gamma>\alpha_{n-1}^{\prime}$.

There is a new notation for $\alpha$ with $\left(\alpha_{n}^{\prime}\right)_{n \in \omega}$ as the fundamental sequence, and the passage from the original notation for $\alpha$ to this new notation can be made effective. (This may not be obvious from the way the definition is given above, but we can look at an ordinal notation and effectively back up to a notation for 0 or the nearest limit ordinal, and once we have done this with $\alpha_{n}$, we have a simple scheme for locating $\alpha_{n}^{\prime}$.)

We are now ready to describe $\mathbf{A}_{\alpha}(S)$, for recursive ordinals $\alpha$. (Actually, we are describing $\mathbf{A}_{a}(S)$ for $a \in O$.)

(1) For any finite even $\alpha=2 m+2$, let $\mathbf{A}_{\alpha}(S)=Z^{m} \cdot \sigma^{*}(S \oplus(\omega-S))$.

(2) For any finite odd $\alpha=2 m+3$, let $\mathbf{A}_{\alpha}(S)=Z^{m} \cdot \sigma^{*}(S)$.

(3) For any limit $\alpha$, let $\left(\alpha_{n}^{\prime}\right)_{n \in \omega}$ be the adjusted fundamental sequence for $\alpha$ (described above), and let $\mathbf{A}_{\alpha}(S)=\sum_{n \in \omega}\left(1+\eta+1+\mathbf{A}_{\alpha_{n}^{\prime}}\left(S_{n}\right)\right)$.

(4) If $\beta$ is a limit ordinal and $\alpha=\beta+1$, then $\mathbf{A}_{\alpha}(S)=\nu^{\alpha}(S \oplus(\omega-S))$.

(5) If $\beta$ is a limit ordinal and $\alpha=\beta+2$, then let $\mathbf{A}_{\alpha}(S)=\nu^{\alpha}(S)$.

(6) If $\beta$ is a limit ordinal and $\alpha=\beta+1+2 k+2=\beta+2 k+3$, then let $\mathbf{A}_{\alpha}(S)=Z^{\beta+k} \cdot \sigma^{*}(S \oplus(\omega-S))$.

(7) If $\beta$ is a limit ordinal and $\alpha=\beta+1+2 k+3=\beta+2 k+4$, then let $\mathbf{A}_{\alpha}(S)=Z^{\beta+k} \cdot \sigma^{*}(S \oplus(\omega-S))$. 
Our next goal in this section is to characterize $\operatorname{Dg}\left(\mathbf{A}_{\alpha}(S)\right)$ for all recursive ordinals $\alpha$. (The set of degrees turns out to be independent of the notation for $\alpha$.$) Having done this, we shall choose appropriate sets S$ so that $\mathbf{A}_{\alpha}(S)$ is an ordering that has $\alpha$ th jump degree and does not have $\beta$ th jump degree for any $\beta<\alpha$. We need some more lemmas. The first of these is related to Lemma 3.1 and will be used for limit ordinals.

Lemma 4.1. Let $\alpha$ be a recursive limit ordinal, with a fundamental sequence $\left(\alpha_{n}\right)_{n \in \omega}$ such that $\alpha_{0} \geq 3$. Suppose that a procedure for encoding a set $S \subseteq \omega \times \omega$ in a sequence of orderings $\left(\mathbf{B}_{n}\left(S_{n}\right)\right)_{n \in \omega}$ satisfies the following conditions:

(a) if $\left(\mathbf{C}_{n}\right)_{n \in \omega}$ is a sequence of orderings such that $\mathbf{C}_{n} \cong B_{n}\left(S_{n}\right)$ for each $n$, then $S_{n} \leq_{T} D\left(\mathbf{C}_{n}\right)^{\left(\alpha_{n}\right)}$ uniformly in $n$,

(b) if $S_{n} \leq_{T} D^{\left(\alpha_{n}\right)}$ uniformly in $n$, then there is a sequence $\left(\mathbf{C}_{n}\right)_{n \in \omega}$ such that $\mathbf{C}_{n} \cong B_{n}\left(S_{n}\right)$ and $D\left(\mathbf{C}_{n}\right) \leq_{T} D$ uniformly in $n$.

(c) for each $n, \mathbf{B}_{n}\left(S_{n}\right)$ has no dense interval.

Now, let $\mathbf{B}(S) \cong \sum_{n \in \omega}\left(1+\eta+1+B_{n}\left(S_{n}\right)\right)$ for some $S \subseteq \omega \times \omega$. Then $\operatorname{Dg}(\mathbf{B}(S))=\left\{\operatorname{deg}(D): S_{n} \leq_{T} D^{\left(\alpha_{n}\right)}\right.$ uniformly in $\left.n\right\}$.

Proof. We must show that for any $D \subseteq \omega$, the following are equivalent:

(1) there exists $\mathbf{B} \cong \mathbf{B}(S)$ such that $D(\mathbf{B}) \leq_{T} D$,

(2) $S_{n} \leq_{T} D^{\left(\alpha_{n}\right)}$ uniformly in $n$.

To show that (1) implies (2), let $\mathbf{B} \cong B(S)$. There is a uniform procedure for finding the endpoints that set off the intervals $\mathbf{C}_{n} \cong \mathbf{B}_{n}\left(S_{n}\right)$, given $n$ and using an oracle for $D(\mathbf{B})^{(3)}$. This is where we use condition (c). Knowing these endpoints and using an oracle for $D(B)^{\left(\alpha_{n}\right)}$, we can tell what is in $D\left(\mathbf{C}_{n}\right)^{\left(\alpha_{n}\right)}$, so by condition (a), we can tell what is in $S_{n}$. To show that (2) implies (1), note that if $S_{n} \leq_{T} D^{\left(\alpha_{n}\right)}$ uniformly in $n$, then by condition (b), there is a sequence of orderings $\left(\mathbf{B}_{n}\right)_{n \in \omega}$ such that $\mathbf{B}_{n} \cong B_{n}\left(S_{n}\right)$ and $D\left(\mathbf{B}_{n}\right) \leq_{T} D$ uniformly in $n$. We can put the $\mathbf{B}_{n}$ 's together to form $\mathbf{B} \cong B(S)$ such that $D(\mathbf{B}) \leq_{T} D$. This completes the proof of the lemma.

The next lemma is used in dealing with the first and second successors of a limit ordinal. The proof uses the ideas from the proof of Lemma 3.2, but it is more complicated.

Lemma 4.2. If $\alpha$ is a recursive limit ordinal, then for any $S \subseteq \omega, \operatorname{Dg}\left(\nu^{\alpha}(S)\right)=$ $\left\{\operatorname{deg}(D): S\right.$ is r.e. in $\left.D^{(\alpha+1)}\right\}$.

Proof. We show that for any $D \subseteq \omega$, the following are equivalent:

(1) there exists $\mathbf{B} \cong \nu^{\alpha}(S)$ such that $D(\mathbf{B}) \leq_{T} D$,

(2) $S$ is r.e. in $D^{(\alpha+1)}$.

Let $\mathbf{B} \cong \nu^{\alpha}(S)$. Recall the equivalence relations $\sim_{\beta}$ on $\mathbf{B}$. Let $d$ be the function such that $d(0)=2, d(\beta+1)=d(\beta)+2$, and for limit $\beta, d(\beta)=$ $\sup \{d(\gamma): \gamma<\beta\}+1=\beta+1$. (We have ordinal notations in mind here, and the function $d$ actually takes $O$ effectively to $O$.) For recursive ordinals $\beta, \sim_{\beta}$ 
is uniformly recursive in $D(\mathbf{B})^{(\alpha(\beta))}$. Now, to see why (1) implies (2), note that $r \in S$ iff there is some $x$, located in a maximal discrete set of size $r+1$, such that for each $\beta<\alpha$, there exists $y>x$ such that $y \sim_{\beta+1} x$ but not $y \sim_{\beta} x$.

The proof that (2) implies (1) uses workers. For each $\beta \leq \alpha+1$, we have Worker $\beta$ equipped with an oracle for $D^{(\beta)}$. A given worker will respond to instructions from the worker immediately above him and may also have to respond to instructions from infinitely many different limit workers. The construction proceeds in infinitely many stages; each worker does something at stage $s$ for each $s \in \omega$. Suppose that $\gamma$ is a limit ordinal, with fundamental sequence $\left(\gamma_{k}\right)_{k \in \omega}$. At stage $s$, Worker $\gamma$ limits his use of the oracle for $D^{(\gamma)}$. He asks questions about $D^{\left(\gamma_{k}\right)}$ only for $k \leq s$. This means that if $\delta \geq \gamma_{k}$, Worker $\delta$ can figure out exactly what Worker $\gamma$ did at stage $k$. (Of course, if $\delta$ is a limit ordinal, Worker $\delta$ may have to wait to ask certain questions of his own oracle.) If $\gamma$ is a successor ordinal, then Worker $\gamma$ can ask whatever questions he likes of his oracle at any stage.

Let $f$ be an enumeration of $S$ such that $f \leq_{T} D^{(\alpha+1)}$. At stage $s$, Worker $\alpha+1$ computes $f(s)$. This is his only job. Worker $\alpha$ guesses values of $f$. We shall describe the guessing process. For any successor ordinal $\gamma=\xi+1$, we have an approximation $D_{s}^{(\gamma)}$ of $D^{(\gamma)}$ consisting of those $e$ such that there is a halting computation of $\varphi_{e}^{D^{(\xi)}}(e)$ consisting of at most $s$ steps and using oracle information available to Worker $\xi$ at stage $s$. Note that if $e \in D^{(\gamma)}$, then $e \in D_{s}^{(\gamma)}$ for all sufficiently large $s$, and if $e \notin D^{(\gamma)}$, then $e \notin D_{s}^{(\gamma)}$ for all $s$. Suppose $f=\varphi_{e}^{D^{(n+1)}}$. At stage $s$, Worker $\alpha$ has a reasonable guess at $f(k)$ if there is a halting computation of $\varphi_{e}^{D_{s}^{(s+1)}}(k)$ consisting of at most $s$ steps. Let $f^{*}(k, n)$ be this guess at $f(k)$, if it exists. By assigning garbage values as needed, we can make the function $f^{*}$ total. The important features of $f^{*}$ are (1) for each $k, \lim _{n \rightarrow \omega} f^{*}(k, n)=f(k)$, and (2) the functions $f_{n}$ such that $f_{n}(k)=f^{*}(k, n)$ are uniformly recursive in $d^{\left(\alpha_{n}\right)}$.

The model B will consist of densely many chains of the forms (a) $r+1+$ $\sum_{1 \leq \xi \leq \beta} Z^{\xi}$, for $1 \leq \beta<\alpha, r \in \omega$, and (b) $r+1+\sum_{1 \leq \xi<\alpha} Z^{\xi}$, for $r \in S$. As in the proof of Lemma 3.2, some of the model is prefabricated. At stage 0, all of the chains will have form (a). Some of these will be extended later so that they end up having form (b). We shall give a finer classification of the chains of form (a). A chain $r+1+\sum_{1 \leq \xi<\beta} Z^{\xi}$ obviously has parameters $r$ and $\beta$ associated with it. We may classify such a chain as $(r, \beta)$-NE, meaning that the chain will not be extended under any circumstances. Or, we may classify the chain as $(r, \beta)$-E $\mid f(k)$, for some $k \in \omega$. We use the latter classification only for certain $\beta$ 's: those such that for some $N, \alpha_{N} \geq 1$ and either $\alpha_{N}<\omega$ and $\beta=\alpha_{N}+1$ or else $\alpha_{N} \geq \omega$ and $\beta=\alpha_{N}$. Every chain classified as $(r, \beta)$-E $\mid f(k)$ will be extended at least a little. The chain will end up with form (b) if $f(k)=r$ and $\beta$ is sufficiently large.

To each rational number $q$, we assign some type of chain (of form (a)). We 
do this in such a way that for each type, the set of rationals to which it is assigned is dense. We also assign to each rational $q$ an infinite set $B_{q}$ of constants. The sets $B_{q}$ are disjoint, and the universe of $\mathbf{B}$ will be $\bigcup\left\{B_{q}: q \in Q\right\}$. If $q, r \in Q$ and $q<r$ (in the usual ordering of the rationals), then the elements of $B_{q}$ will precede those of $B_{r}$ in the ordering of $\mathbf{B}$. If $q$ is assigned type $(r, \beta)$-NE, then $B_{q}$ is arranged in a predetermined way to form a copy of $r+1+\sum_{1 \leq \xi<\beta} Z^{\xi}$. If $q$ is assigned type $(r, \beta)$-E $\mid f(k)$, then half of the constants from $B_{q}$ are arranged in a predetermined way to form a copy of $r+1+\sum_{1 \leq \xi<\beta} Z^{\xi}$. (This ordering is infinite since $\beta \geq 2$.) Let $B_{q}^{*}$ be the remaining half of $B_{q}$.

What we have described above-the assignment of types and constants to rationals, the predetermined ordering of constants in some $B_{q}$ 's and first halves of other $B_{q}$ 's-is all supposed to be recursive. It is the ordering of the remaining sets $B_{q}^{*}$ that depends on $S$. We shall focus our attention on a single $q$ which has been assigned type $(r, \beta)-\mathrm{E} f(k)$. There is a predetermined order on the first half of $B_{q}$. This is a copy of $r+1+\sum_{1 \leq \xi<\beta} Z^{\xi}$ if $\beta=\alpha_{N} \geq \omega$ and of $r+1+\sum_{1 \leq k<\beta} Z^{k+1}$ if $\beta=\alpha_{N}+1<\omega$.

There are three cases to consider.

Case 1. $f^{*}(k, n)=r$ for all $n \geq N$.

Case 2. $f^{*}(k, N) \neq r$.

Case 3. $f^{*}(k, N)=r$, but for some first $M>N, f^{*}(k, M) \neq r$.

In Case $1, B_{q}^{*}$ will end up as a copy of $\sum_{\beta \leq \xi<\alpha} Z^{\xi}$, so that the whole of $B_{q}$ will be a copy of $r+1+\sum_{1 \leq \xi<\alpha} Z^{\xi}$. In Case $2, B_{q}^{*}$ will be a copy of $Z^{\beta}$. In Case 3, $B_{q}^{*}$ will be a copy of $\sum_{\beta \leq \xi<\gamma} Z^{\xi}$, where $\gamma$ is $\alpha_{M}$, if $\alpha_{m} \geq \omega$ and $\alpha_{M}+1$ if $\alpha_{M}<\omega$.

Worker 0 is responsible for copies of $Z$, and in general, if $n<\omega$, Worker $n$ is responsible for copies of $Z^{n+1}$. If $\omega \leq \gamma<\alpha$, then Worker $\gamma$ is responsible for copies of $Z^{\gamma}$. From now on, we shall use the term $\gamma$-block to mean a copy of $Z^{\gamma+1}$ if $\gamma<\omega$ and a copy of $Z^{\gamma}$ if $\gamma \geq \omega$. Then for all $\gamma<\alpha$, we can say that Worker $\gamma$ is responsible for $\gamma$-blocks. At stage $s$, Worker $\gamma$ has a picture of the construction so far. For $\gamma=0$, Worker $\gamma$ 's picture consists of finitely many 0-blocks, with finitely many constants from $B_{q}^{*}$ located within the blocks. For $\gamma>0$, the picture consists of finitely many $\delta$-blocks for $\delta \leq \gamma$, with some of the smaller blocks located inside larger ones. We shall describe the picture in more detail later.

In general, at stage $s$, Worker $\gamma$ first revises his picture. Then if $\gamma>0$, Worker $\gamma$ may give a command. If $\gamma=\delta+1$, a command asks Worker $\delta$ for a new $\delta$-block to help flesh out one of the $\gamma$-blocks in Worker $\gamma$ 's picture. If $\gamma$ is a limit ordinal, a command may ask the lower workers to help flesh out a $\gamma$-block (by building blocks which will fit together) or to destroy an existing 
$\gamma$-block (by stowing various pieces of it in other blocks). Worker 0 does not give commands. After revising his picture, he adds new constants to the 0-blocks.

We need a schedule for the workers to give and respond to commands. We fix a recursive list of pairs in $\alpha \times \omega$ such that if $i<j$, then $(\delta, i)$ comes before $(\delta, j)$. (Of course, we are really thinking of ordinal notations here.) The pair $(\delta, i)$ will represent the $i$ th command of Worker $\delta$. This command may not be given at stage $i$; in general, it will be given at a much later stage. When we say that one command is earlier than another (where the commands may be given by different workers), what we mean is that the pair corresponding to the first command comes before the pair corresponding to the second command on the master list of pairs.

The picture that Worker $\gamma$ has at stage $s$ involves information from the commands that Worker $\gamma$ can read by stage $s$, among those associated with the first $s$ pairs on the list. If Worker $\gamma$ has done anything nontrivial in the construction, then his picture includes an initial $\gamma$-block and possibly further $\gamma$ blocks created in responding to commands from above. (There may be mistakes in the picture because of wrong guesses at commands by Worker $\gamma+1$.) For $\gamma>0$, if Worker $\gamma$ gives a command, to create or destroy a block, the command will say how the lower workers should locate this block in relation to the other blocks in their pictures. It is for this reason that Worker $\gamma$ 's picture must include $\delta$-blocks for $\delta<\gamma$, located as they should be after correct work on earlier commands (possibly within other $\delta^{\prime}$-blocks for $\delta<\delta^{\prime} \leq \gamma$ ).

For each $\gamma \leq \alpha_{N}$, Worker $\gamma$ has just one $\gamma$-block in his picture at stage 0 , all representing a start at the $\alpha_{N}$-block that will be put into the chain. For Worker $\alpha_{N}$, this initial block lies to the left of any $\alpha_{N}$-blocks that may be added later. For $\gamma<\alpha_{N}$, there will be $\gamma$-blocks added to the left and right of the initial one to flesh out this $\alpha_{N}$-block, and there may be other $\gamma$-blocks as well, further to the right. If $f^{*}(k, n)=r$ for all $n \geq N$ such that $\alpha_{N} \leq \gamma$, then Worker $\gamma$ will extend the chain. This puts a leftmost $\gamma$-block in his picture. There will eventually be other $\gamma$-blocks to the right of this if the workers above $\gamma$ are also extending the chain. Before doing anything else, at stage 0 or as soon after that as his oracle information permits, Worker $\gamma$ decides whether to put the first (leftmost) $\gamma$-block into his picture. If he decides not to do this, then he will never have any $\gamma$-blocks, and he will never give any commands.

We must say how Worker $\gamma$ reads commands at stage $s$. He looks at the $\delta$ 's that occur in the first $s$ pairs $(\delta, i)$, and for each of these $\delta$ 's, tries to see what Worker $\delta$ did for the first few stages, keeping track of the commands that were given. For $\delta=\gamma+1$, Worker $\gamma$ can guess what Worker $\delta$ did at stage $k$ for $k<s$, although some of the guesses may be wrong. Suppose that $\delta$ is a limit ordinal, and let $k$ be greatest such that $\delta_{k} \leq \gamma<\delta$. If $\gamma$ is not also a limit ordinal, then Worker $\gamma$ can certainly figure out at stage $s$ what Worker $\delta$ has done up through stage $k$. (If $\gamma$ is a limit ordinal, then Worker $\gamma$ may not be able to do this at stage $s$, although he will be able to do it later.) If 
$\delta<\gamma$, then Worker $\gamma$ can figure out what commands Worker $\delta$ has given by stage $s$ (although if $\gamma$ is a limit ordinal, Worker $\gamma$ may not be able to do this at stage $s$ ). Of course, Worker $\gamma$ knows at stage $s$ what commands he himself has given at earlier stages.

Workers $\gamma$ revises his picture so that it reflects an initial sequence of commands among those that he can read. He may read a new command given by a worker below and incorporate that in his picture, or he may read a command (new or not yet responded to correctly) from a worker above. He will not respond to a command unless he has taken care of all of the earlier commands that he will ever be able to read. He will not respond to a new command if he finds a mistake in his previous work (because of a wrong guess at some command).

Suppose that it is appropriate to respond to a command, say the one associated with $(\delta, i)$. If $\delta=\gamma+1$, then Worker $\delta$ 's $i$ th command must say to start a $\gamma$-block, in a certain place relative to other blocks. When Worker $\gamma$ carries out the command, he puts a new $\gamma$-block in the right place in his picture. Suppose instead that $\delta$ is a limit ordinal, $\delta>\gamma, \delta_{k} \leq \gamma$, and Worker $\delta$ 's $i$ th command was given at stage $k$. The command may say to help start a $\delta$-block. Worker $\gamma$ responds by putting a new $\gamma$-block in the proper place in his picture. (For other $\gamma^{\prime}$ such that $\delta_{k} \leq \gamma^{\prime}<\delta$, Worker $\gamma^{\prime}$ does the same, so when Worker $\gamma^{\prime}$ later issues commands to try to flesh out his $\gamma^{\prime}$-block, Worker $\gamma$ locates the resulting smaller blocks within his $\gamma$-block.) For a limit ordinal $\delta$, Worker $\delta$ 's $i$ th command may say to destroy the block created by an earlier command. Worker $\gamma$ responds to a command of this type by combining the unwanted block with the adjacent $\gamma$-block on the left.

Worker $\gamma$ tries to make sure that the blocks are eventually fleshed out. For Worker 0 , what this means is adding constants at the beginning and end of each 0-block currently in the picture, at the end of each stage. For $\gamma=\xi+1$, Worker $\gamma$ gives a command to Worker $\xi$, at various times when he is allowed to, asking for a new $\xi$-block at the beginning or end of a $\gamma$-block. For $\gamma$ a limit ordinal, Worker $\gamma$ gives a single command to help build a $\gamma$-block. If for all sufficiently large $\delta<\gamma$, Worker $\delta$ creates a $\delta$-block, and all of the blocks are lined up, this is enough. The successor $\delta$ 's will give enough commands to flesh out the $\gamma$-block. Worker $\gamma$ cannot give a command at stage $s$ unless he has read all of the earlier commands that he can ever expect to and has located in his picture the blocks created by himself and the workers below in response to these commands. Worker $\gamma$ will wait if the workers below have not by stage $s$ finished their work on the earlier command.

Now, let us see how Worker $\gamma$ corrects his mistakes. At stage $k$, Worker $\gamma$ may have guessed wrong some command of Worker $\gamma+1$, and he may have carried out the command as guessed. Suppose that Worker $\gamma$ discovers the error at stage $s$. He would like to start back at stage $k$ and do things over from that point, but there are difficulties in doing this. If $\gamma>0$, Worker $\gamma$ may have given several commands between stage $k$ and stage $s$. He now starts 
going through them in reverse order, issuing new commands in some cases to undo what the old commands accomplished. He will not try to carry out any new commands until his picture and the sequence of commands he has given are consistent with the picture of the $\gamma$-blocks at the beginning of stage $k$.

Worker 0 does not issue commands. However, when he discovers an error, he may have extra constants to deal with. Any constants that were introduced since stage $k$ and put into 0-blocks that did not exist at stage $k$ must be reassigned to 0 -blocks that did exist. Backing up one step at a time, Worker 0 takes the constants from a 0 -block that was created at a stage between $k$ and $s$ and assigns them to the nearest 0 -block on the left. If $\gamma=\xi+1$, then Worker $\gamma$ gets rid of a $\gamma$-block created at a stage between $k$ and $s$ by assigning the $\xi$-blocks from the unwanted $\gamma$-block to the nearest $\gamma$-block on the left. The commands that Worker $\gamma$ has given still stand. Worker $\xi$ never hears about Worker $\gamma$ 's error.

Let $\gamma$ be a limit ordinal. Suppose that Worker $\gamma$ has discovered an error and needs to destroy a block that was created in response to his $i$ th command. He gives a new command, his $k$ th, say, to destroy the block associated with $(\gamma, i)$. For all sufficiently large $\delta<\gamma$, Worker $\delta$ will have created a $\delta$-block in response to $(\gamma, i)$ and, for some of these $\delta$ 's, Worker $\delta$ may have given commands $(\delta, j)$, to start fleshing out his $\delta$-block. There are only finitely many pairs $(\delta, j)$ between $(\gamma, i)$ and $(\gamma, k)$. Therefore, for sufficiently large $\delta<\gamma$, there has been no fleshing out by the workers between $\delta$ and $\gamma$. When Worker $\delta$ responds to $(\gamma, k)$ by combining his $\delta$-block with an adjacent one, this will lie outside the unwanted $\gamma$-block. If $\gamma$ is a limit ordinal, Worker $\gamma$ may give a command to cancel a $\gamma$-block and then find that this was a mistake. When this happens, Worker $\gamma$ starts a new block instead of trying to reinstate the old one.

It is fairly easy to show, by induction on the list of commands, that each command eventually gets a correct response, and that all workers who can read the command (and either respond themselves or lie above workers who respond) locate the resulting block in their pictures. From this, it follows that the chain is extended exactly as planned. Those workers who decide at the beginning to extend the chain introduce blocks that are eventually fleshed out by the workers below. All blocks and constants introduced in work that is incorrect are eventually incorporated into blocks that are actually needed for the construction. There is no circularity. What Worker $\gamma$ does at a given stage $s$, when his next command will be his $j$ th, depends on what is done by Worker $\delta$ for $\delta<\gamma$ at stage $k$ for $k \leq s$ and by Worker $\delta$ for $\delta \geq \gamma$ at stage $k$ for $k<s$. Actually, only finitely many of the workers $\delta>\gamma$ are involved-the ones such that there is a pair $(\delta, i)$ before $(\gamma, j)$. this completes our discussion of the proof of Lemma 4.2 .

Lemma 4.2 is true uniformly, as is Lemma 4.3 below. Lemma 4.3 is related to Lemma 2.2. We shall use Lemma 4.2 in conjunction with Lemmas 2.1 and 
2.2 to analyze the orderings $\mathbf{A}_{\beta}(S)$ in case $\beta$ is infinite but is not a limit ordinal or the first or second successor of one.

Lemma 4.3. For any ordering $\mathbf{A}$ and any recursive limit ordinal $\alpha, \operatorname{Dg}\left(Z^{\alpha} \cdot \mathbf{A}\right)=$ $\left\{\mathbf{d}: \mathbf{d}^{(\alpha+1)} \in \operatorname{Dg}(\mathbf{A})\right\}$.

Proof. We must show that for any $D \subseteq \omega$, the following are equivalent:

(1) there exists $\mathbf{B} \cong Z^{\alpha} \cdot \mathbf{A}$ such that $D(\mathbf{B}) \leq_{T} D$,

(2) there exists $\mathbf{C} \cong \mathbf{A}$ such that $D(\mathbf{C}) \leq_{T} D^{(\alpha+1)}$.

The fact that (1) implies (2) follows from the fact that for a limit ordinal $\alpha$, the relation $\sim_{\alpha}$ on $\mathbf{B}$ is recursive in $D(\mathbf{B})^{(\alpha+1)}$. The proof that (2) implies (1) involves a construction by workers which is like that in the proof of Lemma 4.2. We shall only sketch the proof. For each $\beta \leq \alpha+1$, we have Worker $\beta$ equipped with an oracle for $D^{(\beta)}$. At stage $s$, Worker $\alpha+1$ computes the ordering of the first $s+1$ elements of $\mathbf{C}$, which he writes down as $o_{s}$. At stage $s$, Worker $\alpha$ guesses $o_{k}$ for $k \leq s$ and acts on the first $o_{k}$ such that either the current guess differs from the previous guess or else $o_{k-1}$ was acted on at the previous stage. Worker $\alpha$ issues a command $i_{s}$ at stage $s$. This command says either to help start a new $\alpha$-block or to cancel an $\alpha$-block requested in an earlier command. The sequence of commands is like the one given by Worker 1 in the proof of Lemma 2.2. The sequence has the feature that if the workers below succeed in carrying it out, the result will be an ordering that is isomorphic to $Z^{\alpha} \cdot \mathbf{A}$. The proof of Lemma 4.2 actually shows that any sequence of commands of this sort can be carried out. It follows that there is an ordering $\mathbf{B}$ such that $\mathbf{B} \cong Z^{\alpha} \cdot \mathbf{A}$ and $D(\mathbf{B}) \leq_{T} D$. This completes our sketch of the proof of Lemma 4.3 .

The next lemma combines the results from Lemmas 4.3 and 2.2 to characterize $\operatorname{Dg}\left(Z^{\beta} \cdot \mathbf{A}\right)$ in terms of $\operatorname{Dg}(\mathbf{A})$ for an arbitrary infinite recursive ordinal $\beta$. (We dealt with finite $\beta$ in $\S 2$.)

Lemma 4.4. For any ordering $\mathbf{A}$, any recursive limit ordinal $\alpha$, and any $n \in \omega$, $\operatorname{Dg}\left(Z^{(\alpha+n)} \cdot \mathbf{A}\right)=\left\{\mathbf{d}: \mathbf{d}^{(\alpha+1+2 n)} \in \operatorname{Dg}(\mathbf{A})\right\}$.

Proof. We must show that for any $D \subseteq \omega$, the following are equivalent:

(1) there exists $\mathbf{B} \cong Z^{\alpha+n} \cdot \mathbf{A}$ such that $D(\mathbf{B}) \leq_{T} D$,

(2) there exists $\mathbf{C} \cong \mathbf{A}$ such that $D(\mathbf{C}) \leq_{T} D^{(\alpha+1+2 n)}$.

Note that $Z^{\alpha+n} \cdot \mathbf{A} \cong Z^{\alpha} \cdot\left(Z^{n} \cdot \mathbf{A}\right)$. Then the result follows easily from Lemmas 2.2 and 4.3 .

Recall from the beginning of this section the general method for encoding a set $S$ in orderings $\mathrm{A}_{\alpha}(S)$. We are now in a position to characterize the sets $\operatorname{Dg}\left(\mathbf{A}_{\alpha}(S)\right)$ for all recursive ordinals $\alpha$.

Lemma 4.5. Let $\alpha$ be a recursive ordinal. Then

(1) for finite even $\alpha=2 m+2, \operatorname{Dg}\left(\mathbf{A}_{\alpha}\right)=\left\{\operatorname{deg}(D): S \leq_{T} D^{(\alpha)}\right\}$,

(2) for finite odd $\alpha=2 m+3, \operatorname{Dg}\left(\mathbf{A}_{\alpha}\right)=\left\{\operatorname{deg}(D): S\right.$ is r.e. in $\left.D^{(\alpha)}\right\}$, 
(3) for limit $\alpha$, if $\left(\alpha_{n}^{\prime}\right)_{n \in \omega}$ is the adjusted fundamental sequence described above, then $\operatorname{Dg}\left(\mathbf{A}_{\alpha}\right)=\left\{\operatorname{deg}(D): S_{n} \leq_{T} D^{\left(\alpha_{n}^{\prime}\right)}\right.$ uniformly in $\left.n\right\}$,

(4) for $\alpha=\beta+2 k+1$, where $\beta$ is a limit ordinal and $k \in \omega, \operatorname{Dg}\left(\mathbf{A}_{\alpha}\right)=$ $\left\{\operatorname{deg}(D): S \leq_{T} D^{(\alpha)}\right\}$,

(5) for $\alpha=\beta+2 k+2$, where $\beta$ is a limit ordinal and $k \in \omega, \operatorname{Dg}\left(\mathbf{A}_{\alpha}\right)=$ $\left\{\operatorname{deg}(D): S\right.$ is r.e. in $\left.D^{(\alpha)}\right\}$.

The proof of Lemma 4.5 consists of putting together the various lemmas given previously. We get (1) and (2) from Lemma 2.3(a) and (b), respectively. We get (3) from Lemma 4.1. (The hypothesis that the orderings being combined have no dense subintervals is satisfied because none of the ordinals $\alpha_{n}^{\prime}$ in the adjusted fundamental sequence will be limit ordinals.) We get (4) and (5) for $k=0$ from Lemma 4.2. For $k>0$, we apply Lemmas 4.4 and 2.1 instead of Lemma 4.2.

The following theorem is the main result of the paper.

Theorem 4.6. For any recursive ordinal $\alpha \geq 2$, there is an ordering $\mathbf{A}_{\alpha}$ with $\alpha$ th jump degree and not $\beta$ th for $\beta<\alpha$. Moreover, for any $\mathbf{d}>\mathbf{0}^{(\alpha)}$, we can make $\mathbf{A}_{\alpha}$ have ath jump degree $\mathbf{d}$.

Proof. We make use of the classification of recursive ordinals from Lemma 4.5. If $\alpha$ is as in clause (1) or clause (4), then $\alpha$ is the successor of some $\gamma$. By Lemma 1.1, there exist sets $S$ and $D_{0}$ such that $S$ has degree d and $D_{0}^{(\alpha)} \equiv_{T} S$. Then $S$ is in the set $\left\{D^{(\alpha)}: S \leq_{T} D^{(\alpha)}\right\}$ and has least degree among elements of the set, so $\mathbf{A}_{\alpha}(S)$ has $\alpha$ th jump degree $\mathbf{d}$. By Lemma 1.3, if $\gamma<\alpha$, then the set $\left\{D^{(\gamma)}: S \leq_{T} D^{(\kappa)}\right\}$ has no element of least degree. From this, it follows that $\mathbf{A}_{\alpha}(S)$ does not have $\gamma$ th jump degree.

If $\alpha$ is as in clause (2) or clause (5), then again $\alpha$ is the successor of some $\gamma$. We choose $S$ such that $S^{(\alpha)}$ has degree d and $S$ is $\alpha$-generic. By Lemma 1.1 , we have $S^{(\alpha)} \equiv_{T} 0^{(\alpha)} \oplus S$. By Lemma 1.2, if $\gamma<\alpha$, then the set $\left\{D^{(\gamma)}: S\right.$ is r.e. in $\left.D^{(\gamma)}\right\}$ has no element of least degree. Therefore, $\mathbf{A}_{\alpha}(S)$ has $\alpha$ th jump degree $\mathbf{d}$, and for $\gamma<\alpha, \mathbf{A}_{\alpha}(S)$ does not have $\gamma$ th jump degree. If $\alpha$ is as in clause (3), then we choose $S \subseteq \omega \times \omega$ to be $\alpha$-generic with $S^{(\alpha)}$ of degree d. By Lemma 1.1, we have $S^{(\alpha)} \equiv_{T} 0^{(\alpha)} \oplus S$, so $\mathbf{A}_{\alpha}(S)$ has $\alpha$ th jump degree $\mathbf{d}$. Genericity implies that for $\beta<\alpha, S_{k+1}$ is not recursive in $\left(S_{0} \oplus \cdots \oplus S_{k}\right)^{(\beta)}$ for any $k$. By Lemma 1.4 , there is no set of least degree in $\left\{D^{(\beta)}: S_{n} \leq_{T} D^{\left(\alpha_{n}^{\prime}\right)}\right.$ uniformly in $n$ \}. Therefore, $\mathbf{A}_{\alpha}(S)$ does not have $\beta$ th jump degree. This completes the discussion.

It follows from Theorem 4.6 that there is no single recursive ordinal $\alpha$ with the property that all orderings have $\alpha$ th jump degree. Worse is true.

Theorem 4.7. There is an ordering that does not have ath jump degree for any recursive $\alpha$.

Proof sketch. Consider the ordering $\omega_{1}^{C K}$. It is well known that no copy of $\omega_{1}^{C K}$ is hyperarithmetic. Using a special kind of forcing, which is discussed in 
detail in [AKMS], we can show that for any recursive ordinal $\alpha$ and any set $S \subseteq \omega$, if $S \leq_{T} D(\mathbf{A})^{(\alpha)}$ for all $\mathbf{A} \cong \omega_{1}^{C K}$, then $S \leq_{T} 0^{(\alpha)}$. Hence, there is no $\mathbf{B} \cong \omega_{1}^{C K}$ such that $D(\mathbf{B})^{(\alpha)} \leq_{T} D(\mathbf{A})^{(\alpha)}$ for all $\mathbf{A} \cong \omega_{1}^{C K}$.

We close with a pair of questions:

1. For a recursive ordinal $\alpha$, is there an ordering $\mathbf{A}_{\alpha}$ that has $\alpha$ th jump degree $0^{(\alpha)}$ and does not have $\beta$ th jump degree for any $\beta<\alpha$ ?

2. For a recursive ordinal $\alpha$, does there exist an ordering $\mathbf{A}_{\alpha}$ such that $D\left(\mathbf{A}_{\alpha}\right) \equiv_{T} 0^{(\alpha)}$ and for $0 \leq \beta<\alpha$, there is no $\mathbf{B} \cong \mathbf{A}_{\alpha}$ such that $D(\mathbf{B})^{(\beta)} \equiv_{T}$ $0^{(\beta)}$ ?

A negative answer to the second question would obviously imply a negative answer to the first question. However, there is some reason to guess that the answer is positive for both. The result of Jockusch and Soare [JS] gives a positive answer to the second question for $\alpha=1$. Combining this with the fact (from Richter [Ri]) that the only possible degree for an ordering is $\mathbf{0}$, we get a positive answer to the first question also for $\alpha=1$.

\section{REFERENCES}

[A] C. J. Ash, Recursive labelling systems and stability of recursive structures in hyperarithmetical degrees, Trans. Amer. Math. Soc. 298 (1986), 497-514. Correction, 310 (1988), 851.

[AK] C. J. Ash and J. F. Knight, Pairs of structures, Ann. Pure Appl. Logic (to appear).

[AKMS] C. J. Ash, J. F. Knight, M. Manasse, and T. Slaman, Generic copies of countable structures, Ann. Pure Appl. Logic 42 (1989), 195-205.

[H] L. Harrington, Building nonstandard models of Peano arithmetic, handwritten notes, 1979.

[JS] C. G. Jockusch and R. I. Soare, Degrees of linear orderings not isomorphic to recurisve ones, preprint.

[K $\left.\mathrm{K}_{1}\right] \quad$ J. F. Knight, Effective construction of models, Logic Colloquium '84 (J. B. Paris, A. J. Wilkie, and G. M. Wilmers, eds.), North-Holland, 1986, pp. 105-119.

$\left[\mathrm{K}_{2}\right]$ _ Degrees coded in jumps of orderings, J. Symbolic Logic 51 (1986), 1034-1042.

$\left[\mathrm{K}_{3}\right] \quad$, Degrees of models with prescribed Scott set, Classification Theory: Proc., Chicago, 1985 (J. Baldwin, ed.), Lecture Notes in Math., vol. 1292, Springer-Verlag, 1988, pp. 182191.

$\left[\mathrm{K}_{4}\right] \_$, A metatheorem on constructions by finitely many workers, J. Symbolic Logic (to appear).

[L] M. Lerman, On recursive linear orderings, Logic year 1979-80 (Univ. of Conn.), Lecture Notes in Math., vol. 859, Springer-Verlag, 1981, pp. 132-142.

[Mac] J. Macintyre, Transfinite extensions of Friedberg's completeness criterion, J. Symbolic Logic 42 (1977), 1-10.

[Mar] D. Marker, Degrees of models of true arithmetic, Proc. of the Herbrand Sympos. (J. Stern, ed.), North-Holland, 1982, pp. 233-242.

[Ri] L. J. Richter, Degrees of structures, J. Symbolic Logic 46 (1981), 723-731.

[Ro] H. Rogers, Theory of recursive functions and effective computability, McGraw-Hill, 1967.

Department of Mathematics, Monash University, Clayton, Victoria, 3168 Australia

Department of Mathematics, University of Illinois, Urbana, Illinois 61801

Department of Mathematics, University of Notre Dame, Notre Dame, Indiana 46556 